\title{
H I-rich dwarf galaxies in the Hydra I cluster
}

\section{Photometric and $\mathrm{HI}$ data $^{\star}$}

\author{
P.-A. Duc ${ }^{1,2}$, P. Papaderos ${ }^{3}$, C. Balkowski ${ }^{4}$, V. Cayatte $^{4}$, T.X. Thuan $^{5}$, and W. van Driel ${ }^{6}$ \\ 1 Institute of Astronomy, Madingley Road, Cambridge, CB3 0HA, UK \\ 2 ESO, Karl-Schwarzschild-Str. 2, D-85748 Garching bei München, Germany \\ 3 Universitäts-Sternwarte, Geismarlandstr. 11, D-37083 Göttingen, Germany \\ ${ }^{4}$ DAEC, URA 173 associée au CNRS et à l'Université Paris 7, Observatoire de Paris, F-92195 Meudon Cedex, France \\ 5 Astronomy Department, University of Virginia, Charlottesville, VA 22903, U.S.A. \\ ${ }^{6}$ Unité Scientifique Nançay, CNRS USR B704, Observatoire de Paris, F-92195 Meudon Cedex, France
}

Received December 7, 1998; accepted March 1, 1999

\begin{abstract}
We present H I, optical and near-infrared photometric data of a sample of $\mathrm{H}$ I-selected dwarf galaxy candidates in the Hydra I cluster. This database has been compiled in order to study environmental effects on dwarf galaxies in clusters, in particular the role of interactions between them and either the intracluster-medium or companion galaxies, hitherto studied mainly in massive galaxies. The atomic gas is supposed to be very sensitive to any external perturbation, as it is more extended than the stellar disk especially in the case of dwarf star-forming galaxies. In that respect, the discovery of a large number of $\mathrm{H}$ I sources presumably associated with dwarf galaxies in a complete VLA survey of the Hydra I cluster by McMahon (1993) is intriguing since low-mass clouds might be very fragile in a cluster environment. In order to understand the origin and survival of these $\mathrm{H}$ I sources, we have carried out a multi-wavelength analysis of their host galaxies, which were all selected on $21 \mathrm{~cm} \mathrm{Hi}$ line widths smaller than $130 \mathrm{~km} \mathrm{~s}^{-1}$. Sixteen out of the 20 candidates in the initial VLA sample have been reobserved with much higher velocity resolution at Nançay. The $\mathrm{H}$ I detection has been confirmed for all of them. From the combined $\mathrm{H}$ I and optical data, 15 objects turn out to be true dwarf galaxies; for one $\mathrm{H}$ I source no optical counterpart was found, and the remaining 4 galaxies are in fact face-on spirals that were therefore rejected in our final analysis. The $\mathrm{HI}$-selected dwarf galaxies show a large diversity in their morphologies and surface brightness profiles. They range from blue compact objects to red low surface brightness galaxies.
\end{abstract}

Send offprint requests to: P.-A. Duc,

e-mail: pduc@ast.cam.ac.uk

* Based partly on observations collected at the European Southern Observatory, La Silla, Chile (ESO Nos. 58.A-0369 and 60.A-0791).
Key words: galaxies: ISM — galaxies: photometry galaxies: dwarf - galaxies: clusters: Hydra I cluster radio lines: galaxies

\section{Introduction}

It is now well known that the evolution of galaxies is largely governed by their environment. Interactions with companion galaxies or with the intergalactic medium may either enhance or inhibit their star formation and perturb their morphology. Such effects have been studied extensively for large galaxies: in particular, the stripping by the intra-cluster medium of atomic gas from cluster spiral galaxies (see review by Kenney 1990) and the more dramatic tidal disruption of disks during galactic collisions (Schweizer 1998) and, in a more general sense, the morphology-density relation that may result from it (Dressler et al. 1997).

However, little is known at present about the role of the environment on the evolution of dwarf galaxies though they represent ideal laboratories to study it, as they are numerous and their low mass should make them very sensitive to any external perturbation. Some preliminary studies have put forward a number of intriguing properties of dwarf galaxies in clusters. Hoffman et al. (1988) found that dwarf galaxies are not more $\mathrm{H}$ I deficient than spirals, while Valluri \& Jog (1990) claimed that the deficiency actually depends on the galaxy size, with dwarfs being the least deficient. These are apparently surprising results, since the H I gas in dwarfs, especially in dwarf irregulars, tends to be relatively more diffuse and extended than in spirals. However how easily the HI will be stripped 


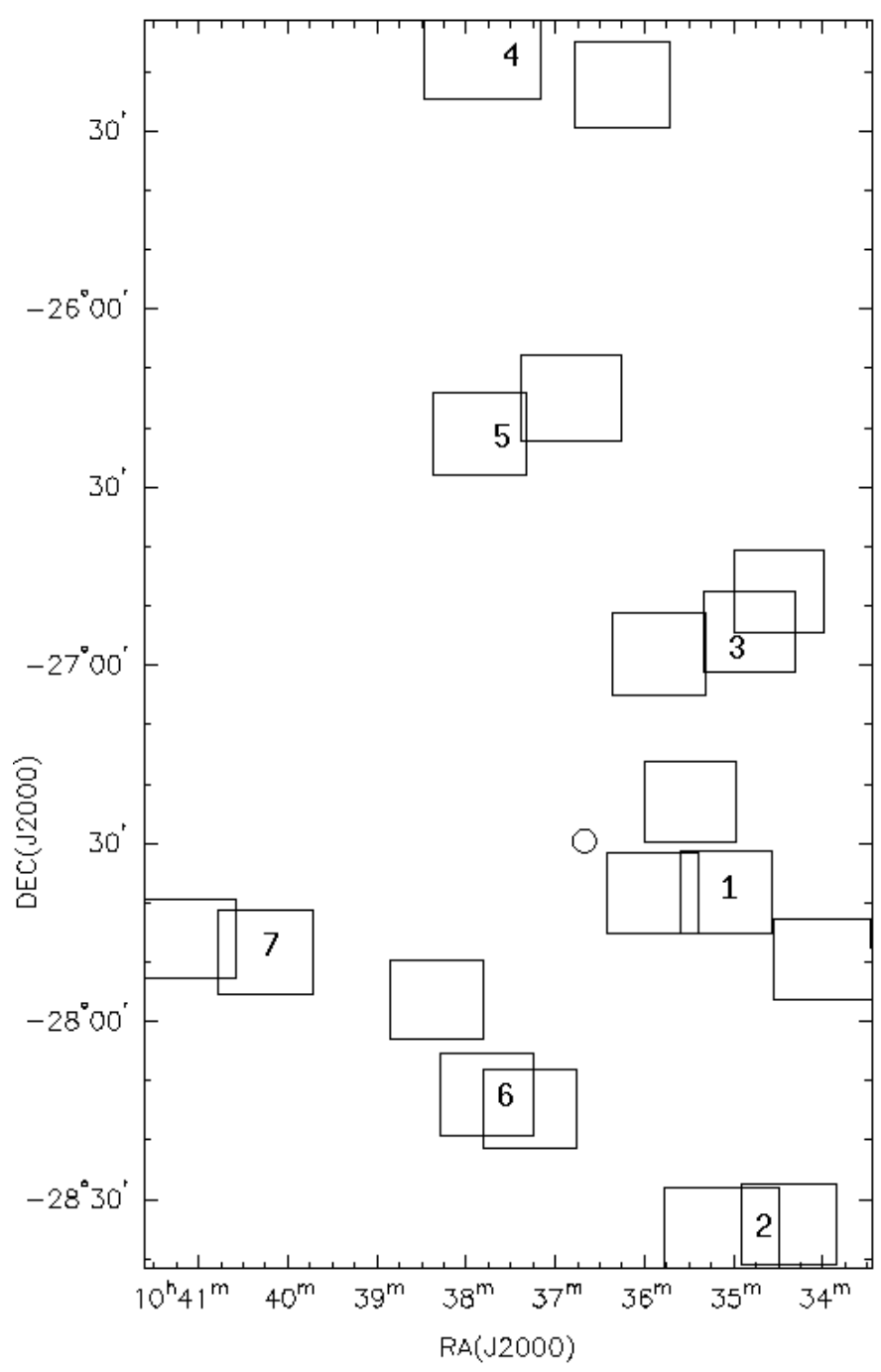

Fig. 1. Locations in the Hydra I cluster of the fields imaged with the DFOSC CCD camera. Each of the 16 rectangles covers the final field of view of the co-added $I$-band images. The final area covered by our imaging survey may thus be easily visualized. The numbers refer to the field ID of the finding charts shown in Figs. 2-8. The circle indicates the location of the cluster core as indicated by the center of the X-ray gas distribution and the position of the giant elliptical NGC 3311

from the galaxy depends on how much it is gravitationally bound to the stellar component and to the dark matter halo which might be the dominant mass.

The complete, three-dimensional coverage made in $\mathrm{H} \mathrm{I}$ with the VLA of two clusters, Hydra (McMahon 1993; Valluri et al. 1999) and Hercules (Dickey 1997), has shown the presence of $\mathrm{HI}$ sources not associated with previously identified massive galaxies. Similar H I sources were also found in VLA fields centered on bright galaxies in two other clusters, Virgo (Cayatte et al. 1990) and Coma (Bravo et al. 1999). The survival of such low-mass H Iclouds in the close environment of stripped cluster spirals, as observed in Hercules, Virgo and Coma, is puzzling.
Numerous surveys of optically selected dwarf galaxies in nearby clusters have already been performed and were not very conclusive as regards the role of the environment: e.g. Bothun et al. (1986); Gallagher \& Hunter (1989), Impey et al. (1988) and Almoznino \& Brosch (1998) in Virgo; Secker et al. (1997) and Trentham (1998) in Coma. However, most of the abovementioned works were mainly based on the study of stellar properties which are a priori less affected by the environment than the gas. When available, the $\mathrm{H}$ I data of these clusters suffer from severe incompleteness. In a cluster as close as Virgo, H I maps have been obtained for only a limited number of galaxies. This lead us to adopt a different approach in the study of environmental effects on dwarf galaxies, i.e., through selection of $\mathrm{H}$ I sources likely to be associated with dwarf galaxies in complete surveys of the atomic gas in clusters, and studies of the properties of their stellar counterparts.

Among the nearby clusters of galaxies, the Hydra I cluster (Abell 1060), at an adopted distance of $45 \mathrm{Mpc}$ $\left(H_{0}=75 \mathrm{~km} \mathrm{~s}^{-1} \mathrm{Mpc}^{-1}\right)$, has the advantage of not being as hugely extended over the sky as Virgo. Hence a quasicomplete radio synthesis $\mathrm{H}$ I survey towards this cluster would be feasible with a reasonable amount of pointings, and has in fact been made by McMahon (1993) at the VLA. Hydra I is classified as an irregular cluster of richness class 1 and morphological type III in the nomenclature of Bautz \& Morgan (1970). This cluster, which appears to have a regular structure in X-ray (Fitchett \& Merritt 1988), is at first sight dynamically relaxed and its large spirals show apparently only moderate $\mathrm{H}$ I deficiencies (Richter \& Huchtmeier 1983). However, various analyses of the dynamics of the cluster indicate evidence of substructures that could explain the lack of on-going gas stripping from the massive galaxies (Fitchett \& Merritt 1988; McMahon et al. 1992 and recently Valluri et al. 1999). A major result of the H I survey of Hydra I by McMahon (1993) is the discovery of numerous H I sources likely to be associated with low-mass galaxies.

Several optical catalogs of galaxies in Hydra have already been compiled by Richter et al. (1982) [hereafter R82], Richter (1987) [R87] - updated in 1989 (Richter 1989), and more recently by Hamabe (1993). However, they include galaxies brighter than $V=17$ only and have therefore missed a lot of dwarf galaxies, in particular, as we will see, most of the optical counterparts of the faint VLA H I sources.

In this paper, we present $\mathrm{HI}$ line spectra and optical/near-infrared photometric data for a sample of $\mathrm{HI}$-selected dwarf galaxies in the Hydra I cluster. The selection criteria are detailed in Sect. 2, while the observations made at Nançay and at ESO are described in Sect. 3. Section 4 deals with the principal results: the identification of the $\mathrm{HI}$ sources, a careful surface brightness and color profiles analysis of their stellar counterparts and a preliminary comparison of the 


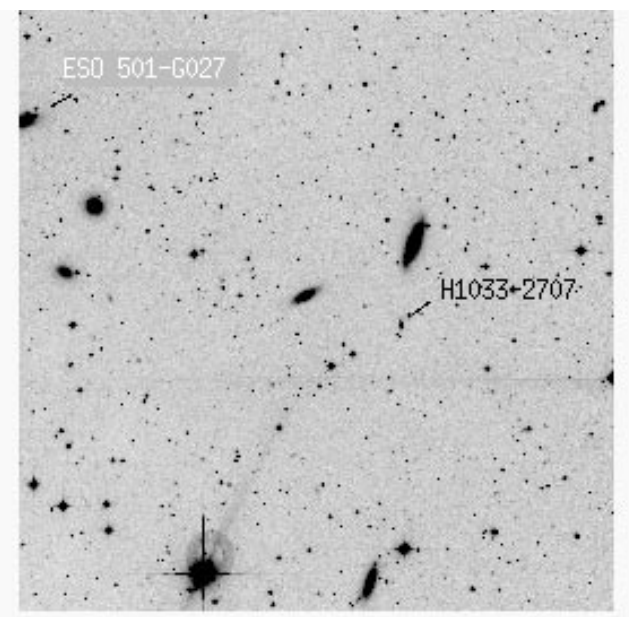

\section{FIELD 1}
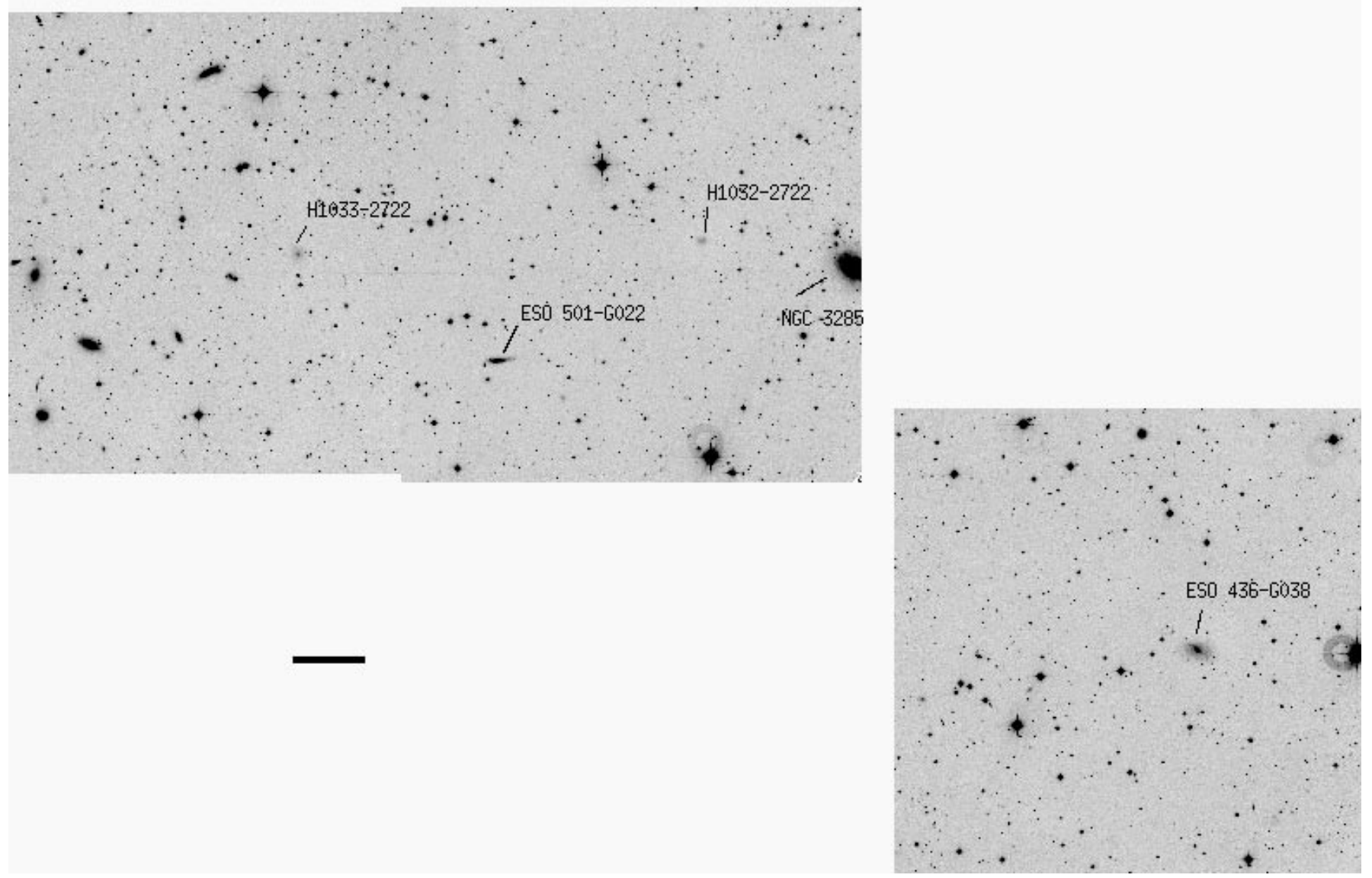

Fig. 2. Identification chart of Field 1. The chart is composed of the co-added DFOSC $I$-band images obtained at the location of Field 1. The galaxies detected in H I with the VLA (McMahon 1993) are identified with black labels. Several H I-poor galaxies that appear to be companions to the H I-detected dwarf galaxies are also indicated with white labels. The bar at the bottom indicates a scale of $2^{\prime}$ or $26 \mathrm{kpc}$ at the adopted distance of Hydra I. North is to the top and East to the left 


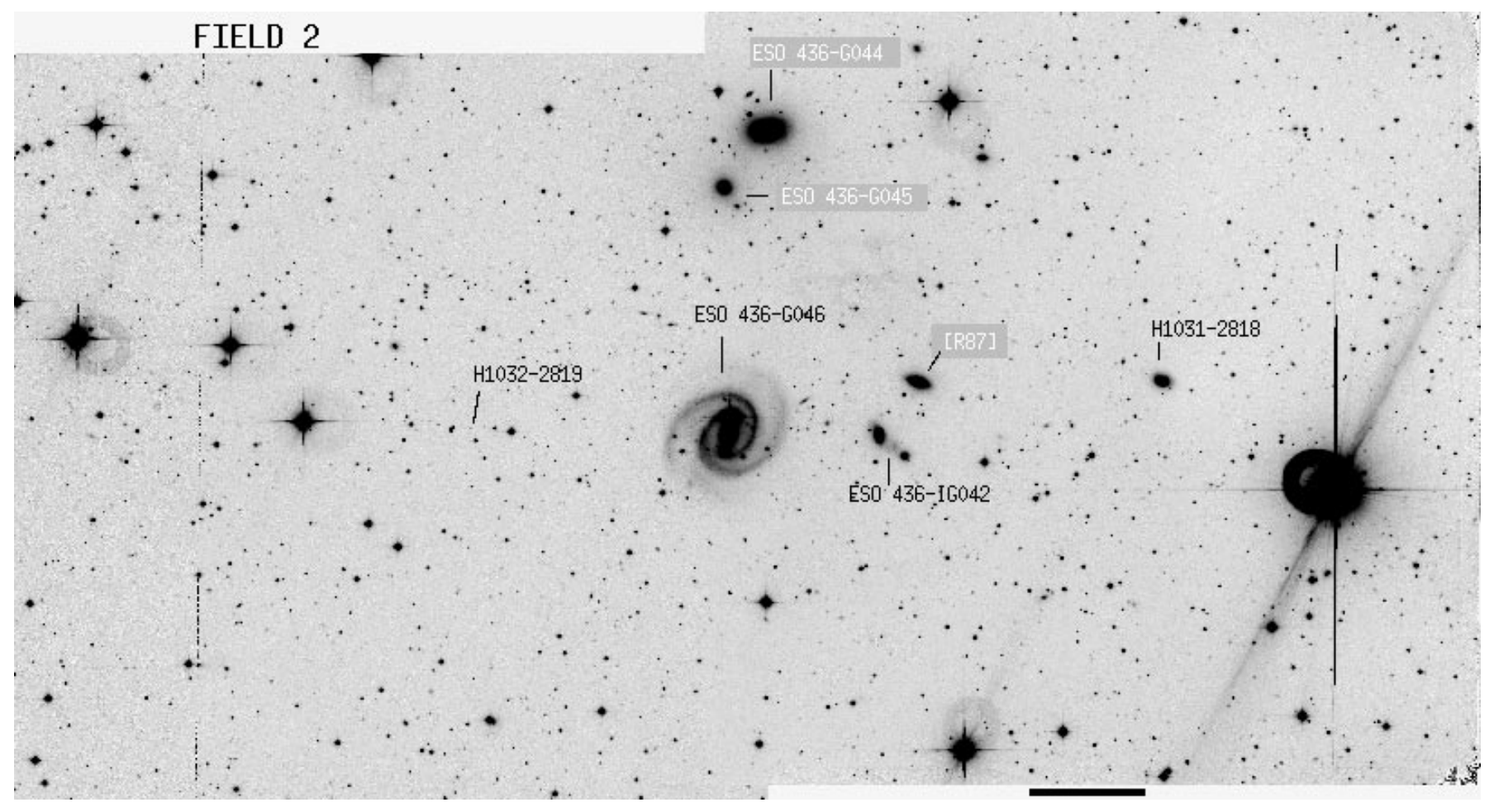

Fig. 3. Identification chart of Field 2 (see Fig. 2 for details)

structural properties of the Hydra dwarfs with other field and cluster dwarf galaxies. Finally, in Sect. 5 notes on individual objects in the sample are presented. Long-slit spectroscopic data obtained in March 1998 with the ESO $3.6 \mathrm{~m}$ telescope will be presented in Paper II (Duc et al., in preparation) whereas the general discussion on the environmental effects on the $\mathrm{HI}$-detected Hydra dwarfs will be the subject of another paper of these series.

\section{The sample selection}

The originality of the present study mainly resides in the selection criteria used and the completeness of our sample. Our H I-selected sample has been extracted from the catalog of H I sources in the Hydra I cluster compiled by McMahon (1993) using VLA mapping. The H I observations are also described in detail by Valluri et al. (1999). McMahon's survey covered the area of the Hydra cluster core with a series of 26 hexagonally positioned pointings. At each pointing the entire radial velocity range of the cluster was covered. Seventy percent of the cluster core volume has thus been imaged. The spatial resolution in the VLA C/D configuration was $45^{\prime \prime}$ and the velocity resolution was $42 \mathrm{~km} \mathrm{~s}^{-1}$. The $5 \sigma$ sensitivity of the observations varied between $2.510^{7} M_{\odot}$ and $1.110^{8} M_{\odot}$ per $42 \mathrm{~km} \mathrm{~s}^{-1}$ wide channel for unresolved objects, with column densities in the range $2.410^{19}-1.310^{20} \mathrm{~cm}^{-2}$ (Valluri et al. 1999).

We initially selected for our catalog all objects with a $21 \mathrm{~cm}$ line width below $130 \mathrm{~km} \mathrm{~s}^{-1}$, as objects with such narrow Hi lines are good dwarf candidates. The rather low velocity resolution of the VLA, $42 \mathrm{~km} \mathrm{~s}^{-1}$, degrades the rigor of this selection criterion, though. Nevertheless, our new $21 \mathrm{~cm}$ line observations obtained at Nançay with a considerably higher velocity resolution, $15 \mathrm{~km} \mathrm{~s}^{-1}$, have confirmed the narrowness of the $\mathrm{H}$ I lines in almost all cases (see Sect. 4.1). Among the $23 \mathrm{HI}$-preselected galaxies, 3 bright face-on spiral galaxies could already be excluded after inspection of their images in the Digital Sky Survey. The coordinates and, when available, the optical identification in the NED and LEDA databases of the 20 objects of our primary list are indicated in Table 1. The original VLA H I data from McMahon (1993), used for our sample selection, are given in Table 2, where it should be noted that her quoted VLA line width is not a strictly measured physical parameter, but only the velocity coverage of the line channels in which a particular object was detected. This is roughly equivalent to the width at $20 \%$ of the peak flux density value.

We will see in Sect. 4 that, based on morphological and new $\mathrm{H}$ I data, 4 galaxies in this list cannot be classified as dwarf galaxies and/or do not meet the velocity width criteria. Moreover, one H I source has no optical counterpart. Therefore our final list includes $15 \mathrm{HI}$-selected objects that we confirmed to be dwarf galaxies. These are the objects listed in the first part of Tables $1-3$. They have integrated $21 \mathrm{~cm}$ line fluxes ranging from $0.16 \mathrm{Jy} \mathrm{km} \mathrm{s}^{-1}$ to $1.36 \mathrm{Jy} \mathrm{km} \mathrm{s}^{-1}$. 
Table 1. Primary list of H I-selected dwarf galaxy candidates in the Hydra cluster

\begin{tabular}{|c|c|c|c|c|}
\hline Name & $\begin{array}{l}\text { Identification } \\
\text { NED/LEDA }\end{array}$ & $\begin{array}{l}\mathrm{RA} \\
\quad(\mathrm{J} 2 \\
\end{array}$ & $\begin{array}{l}\text { DEC } \\
00)^{2}\end{array}$ & Field \\
\hline H1031 - 2818 & PGC 31270 [R87] & $10: 34: 16.6$ & $-28: 34: 04$ & 2 \\
\hline H1031 - 2632 & & $10: 34: 20.7$ & $-26: 47: 31$ & 3 \\
\hline H1032 - 2638 & & $10: 34: 40.5$ & $-26: 54: 33$ & 3 \\
\hline H1032 - 2722 & & $10: 34: 56.1$ & $-27: 38: 25$ & 1 \\
\hline H1033 - 2707 & & $10: 35: 21.7$ & $-27: 23: 27$ & 1 \\
\hline H1033 - 2642 & & $10: 35: 41.1$ & $-26: 58: 21$ & 3 \\
\hline H1033 - 2722 & & $10: 35: 46.8$ & $-27: 38: 50$ & 1 \\
\hline H1034 - 2558 & CTS 1015; M02.13 & $10: 36: 54.8$ & $-26: 14: 27$ & 5 \\
\hline H1034 - 2758 & PGC 31541 [R82] & $10: 37: 19.8$ & $-28: 14: 19$ & 6 \\
\hline H1035 - 2756 & & $10: 37: 38.8$ & $-28: 12: 25$ & 6 \\
\hline H1035 - 2605 & & $10: 37: 41.0$ & $-26: 20: 56$ & 5 \\
\hline H1035 - 2502 & & 10:37:51.3 & $-25: 18: 07$ & 4 \\
\hline H1035 - 2740 & & $10: 38: 11.9$ & $-27: 56: 15$ & 6 \\
\hline H1038 - 2733 & & $10: 40: 26.4$ & $-27: 48: 53$ & 7 \\
\hline H1038 - 2730 & & 10:40:58.8 & $-27: 45: 47$ & 7 \\
\hline H1031 - 2734 & ESO 436- G038 & $10: 33: 53.8$ & $-27: 49: 47$ & 1 \\
\hline H1032 - 2819 & - & 10:35:10.1 & $-28: 34: 56$ & 2 \\
\hline H1033 - 2726 & ESO 501- G022 & $10: 35: 21.7$ & $-27: 41: 45$ & 1 \\
\hline H1033 - 2506 & ESO 501- G032 & $10: 36: 22.2$ & $-25: 22: 35$ & 4 \\
\hline H1034 - 2553 & LEDA 155460 [R82] & $10: 36: 50.8$ & $-26: 09: 18$ & 5 \\
\hline
\end{tabular}

The coordinates correspond to the center of the optical counterparts except for H1032-2819 whose coordinates are taken from the H I catalog of McMahon (1993).

Table 2. H I data of the primary list dwarf galaxy candidates

\begin{tabular}{|c|c|c|c|c|c|c|c|c|}
\hline \multirow[b]{2}{*}{ Name } & \multicolumn{3}{|c|}{$-\mathrm{VLA}-$} & \multicolumn{5}{|c|}{ - Nançay - } \\
\hline & $\begin{array}{c}V_{\mathrm{HI}} \\
\mathrm{km} \mathrm{s}^{-1}\end{array}$ & $\begin{array}{c}\text { Width } \\
\mathrm{km} \mathrm{s}^{-1}\end{array}$ & $\begin{array}{r}\int S \mathrm{~d} v \\
\mathrm{Jy} \mathrm{km} \mathrm{s}^{-1}\end{array}$ & $\begin{array}{c}V_{\mathrm{HI}} \\
\mathrm{km} \mathrm{s}^{-1}\end{array}$ & $\begin{array}{c}W_{50} \\
\mathrm{~km} \mathrm{~s}^{-1}\end{array}$ & $\begin{array}{c}W_{20} \\
\mathrm{~km} \mathrm{~s}^{-1}\end{array}$ & $\underset{\mathrm{Jy} \mathrm{km} \mathrm{s}^{-1}}{\int S \mathrm{~d} v}$ & $\begin{array}{l}\mathrm{rms} \\
\mathrm{mJy}\end{array}$ \\
\hline H1031 - 2818 & 3578 & 84 & $0.31 \pm 0.13$ & 3590 & 87: & 102: & 0.47 & 1.3 \\
\hline H1031 - 2632 & 4297 & $\leq 42$ & $0.28 \pm 0.05$ & 4326 & 68 & 81 & 0.37 & 1.6 \\
\hline H1032 - 2638 & 4509 & 128 & $0.38 \pm 0.07$ & - & - & - & - & - \\
\hline H1032 - 2722 & 3018 & $\leq 42$ & $0.33 \pm 0.09$ & 3021 & 49 & 83 & 0.90 & 2.7 \\
\hline H1033 - 2707 & 3144 & 126 & $0.81 \pm 0.08$ & 3147 & 82 & 116 & 1.20 & 2.9 \\
\hline H1033 - 2642 & 2598 & 126 & $0.64 \pm 0.09$ & 2599 & 88 & 104: & 1.10 & 2.1 \\
\hline H1033 - 2722 & 4764 & 128 & $1.36 \pm 0.13$ & 4747 & 94 & 110 & 1.53 & 2.9 \\
\hline H1034- 2558 & 3634 & 84 & $0.24 \pm 0.09$ & 一 & - & 一 & - & - \\
\hline H1034- 2758 & 3469 & 127 & $0.86 \pm 0.17$ & 3468 & 107 & 136 & 0.96 & 2.7 \\
\hline H1035 - 2756 & 4700 & 85 & $0.80 \pm 0.11$ & 4715 & 55 & 94 & 1.30 & 2.4 \\
\hline H1035 - 2605 & 3719 & 84 & $0.16 \pm 0.04$ & 一 & - & - & - & - \\
\hline H1035 - 2502 & 4040 & 84 & $0.41 \pm 0.08$ & 4008 & 59 & 88 & 0.50 & 2.7 \\
\hline H1035 - 2740 & 2872 & 84 & $0.31 \pm 0.07$ & - & - & - & - & - \\
\hline H1038 - 2733 & 4335 & $\geq 84$ & $\geq 0.97 \pm 0.14$ & 4346 & 57 & 67 & 1.04 & 3.2 \\
\hline H1038 - 2730 & 3996 & 127 & $0.97 \pm 0.21$ & 4037 & 155 & 173 & 1.28 & 2.3 \\
\hline H1031-2734 & 2724 & 126 & $1.22 \pm 0.30$ & 2755 & 186 & 208 & 2.11 & 2.9 \\
\hline H1032 - 2819 & 3304 & $\leq 42$ & $0.44 \pm 0.10$ & 3280 & 95 & 120: & 0.96 & 2.8 \\
\hline H1033 - 2726 & 2892 & $\geq 126$ & $2.00 \pm 0.23$ & 2891 & 168 & 194 & 1.94 & 2.4 \\
\hline H1033 - 2506 & 4019 & 127 & $1.25 \pm 0.17$ & 4018 & 103 & 170 & 2.00 & 2.7 \\
\hline H1034- 2553 & 3655 & 126 & $2.42 \pm 0.17$ & 3651 & 78 & 109 & 3.84 & 3.1 \\
\hline
\end{tabular}

VLA data: from McMahon (1993), whose Width is roughly equivalent to $W_{20}$.

The velocities are heliocentric and in the optical convention. 
Table 3. Total effective exposure times in minutes

\begin{tabular}{lccc}
\hline Name & \multicolumn{3}{c}{ Photometric Band } \\
\cline { 2 - 4 } & $B$ & $I$ & $K^{\prime}$ \\
\hline H1031 - 2818 & 30 & 30 & 18 \\
H1031 - 2632 & 30 & 30 & 25 \\
H1032 - 2638 & 30 & 30 & 39 \\
H1032 - 2722 & 30 & 30 & 48 \\
H1033 - 2707 & 30 & 30 & 35 \\
H1033 - 2642 & 30 & 30 & 16 \\
H1033 - 2722 & 30 & 30 & - \\
H1034-2558 & 30 & 30 & 35 \\
H1034-2758 & 15 & 12 & 20 \\
H1035 - 2756 & 30 & 30 & - \\
H1035-2605 & 15 & 22 & 13 \\
H1035 - 2502 & 30 & 30 & - \\
H1035 - 2740 & 15 & 15 & 13 \\
H1038 - 2733 & 30 & 30 & 5 \\
H1038 - 2730 & 15 & 15 & 60 \\
\hline H1031 - 2734 & 30 & 30 & - \\
H1032 - 2819 & 15 & 22 & - \\
H1033 - 2726 & 30 & 30 & - \\
H1033 - 2506 & 30 & 30 & - \\
H1034 - 2553 & 30 & 30 & - \\
\hline
\end{tabular}

\section{Observations and data reduction}

\subsection{Nançay $H_{I}$ observations}

During the period November 1997 - November 1998 we obtained, with the Nançay decimetric radio telescope 21cm, H I line spectra of 19 Hydra cluster galaxies, including 16 objects from our primary list (see Table 2), as well as 3 spirals close to the sample objects. The remaining 4 VLA sources from our primary list were considered too weak for detection at Nançay, given the data published by McMahon (1993). Separate observations were also made with pointing centers around the $\mathrm{H}$ I source without an optical counterpart, H1032 - 2819, in order to confirm the weak VLA detection.

The Nançay telescope is a meridian transit-type instrument with an effective collecting area of roughly $7000 \mathrm{~m}^{2}$ (equivalent to a $94-\mathrm{m}$ parabolic dish). Due to the elongated geometry of the telescope, at $21-\mathrm{cm}$ wavelength it has a half-power beam width of 3.6 E-W $\times 22^{\prime} \mathrm{N}-\mathrm{S}$ at the declination of the Hydra cluster. Tracking was generally limited to about 45 minutes per source. Typical system temperatures were $\sim 40 \mathrm{~K}$.

We obtained our observations in total power (positionswitching) mode using consecutive pairs of two-minute onand two-minute off-source integrations. Off-source integrations were taken at approximately $20^{\prime} \mathrm{E}$ of the target position. The 1024 channel autocorrelator was divided into two pairs of cross-polarized $(H$ and $V$ ) receiver banks, each with 512 channels and a $6.4 \mathrm{MHz}$ wide bandpass. This yielded a channel spacing of $2.64 \mathrm{~km} \mathrm{~s}^{-1}$, for an effective velocity resolution of $\sim 3.3 \mathrm{~km} \mathrm{~s}^{-1}$ at $21 \mathrm{~cm}$. The center frequencies of the two banks were tuned to the known redshifted $\mathrm{H}$ I frequency of the target, as measured at the VLA by McMahon (1993). Total integration times were about 5 to 6 hours for most galaxies, but shorter for some of the stronger spirals and longer for the faintest objects.

We reduced our H I spectra using the standard Nançay spectral line reduction packages available at the Nançay site. With this software we subtracted baselines (generally third order polynomials), averaged the two receiver polarizations, and applied a declination-dependent conversion factor to convert from units of $T_{\text {sys }}$ to flux density in $\mathrm{mJy}$. The $T_{\mathrm{sys}}$-to-mJy conversion factor is determined via a standard declination-dependent calibration relation established by the Nançay staff through regular monitoring of strong continuum sources. This procedure yields a calibration accuracy of $\sim 10 \%$. In addition, we applied a flux scaling factor of 1.26 to our spectra based on statistical comparisons of recent Nançay data on strong, compact spiral galaxies unresolved by the telescope beam (Matthews et al. 1998) with past observations made at Nançay and elsewhere.

\subsection{Optical imaging}

We carried out optical imaging observations in February 1997 with the Danish 1.5 meter telescope at La Silla observatory. We used the DFOSC optical camera which offers a large field of view of $13.7 \times 13.7$. The detector was a LORAL $2 \mathrm{k} \times 2 \mathrm{k}$ CCD with a pixel size of 0 ". 39 .

Images through the Bessel $B$ and Gunn $I$ filters were obtained for sixteen individual positions in 7 distinct fields of Hydra I containing the $20 \mathrm{H}$ I sources of the primary list. The location of these fields in the cluster are indicated in Fig. 1, the field number of each source is given in Col. 5 of Table 1 and the identification charts of the 7 fields are presented in Figs. 2-8. For most objects, $B$-band observations consisted of two individual exposures of $900 \mathrm{~s}$ each, whereas $I$-band observations consisted of 4 individual exposures of $450 \mathrm{~s}$ each. However, due to time constraints, some fields were observed with a lower total exposure time, as indicated in Table 3 . Offsets of $10^{\prime \prime}$ for the observations in the $B$-band and $30^{\prime \prime}$ for the $I$-band were performed between each exposure. Several standard stars from the fields of Landolt (1992) were observed throughout the nights. Weather conditions were photometric.

Standard data reduction was performed using the CCDRED package in IRAF. A median image had to be produced from adjacent $I$-band images, by which the original images were divided in order to remove the prominent fringes seen in the $I$-band. The reduced frames were then co-added with a shift-and-add method. The flux calibration has been performed from photometric transformation equations, whose coefficients, i.e., zero-points, color and extinction terms, were determined from our standard stars observations. The astrometry of all images has been carried out with guide stars from the USNO catalog, queried 


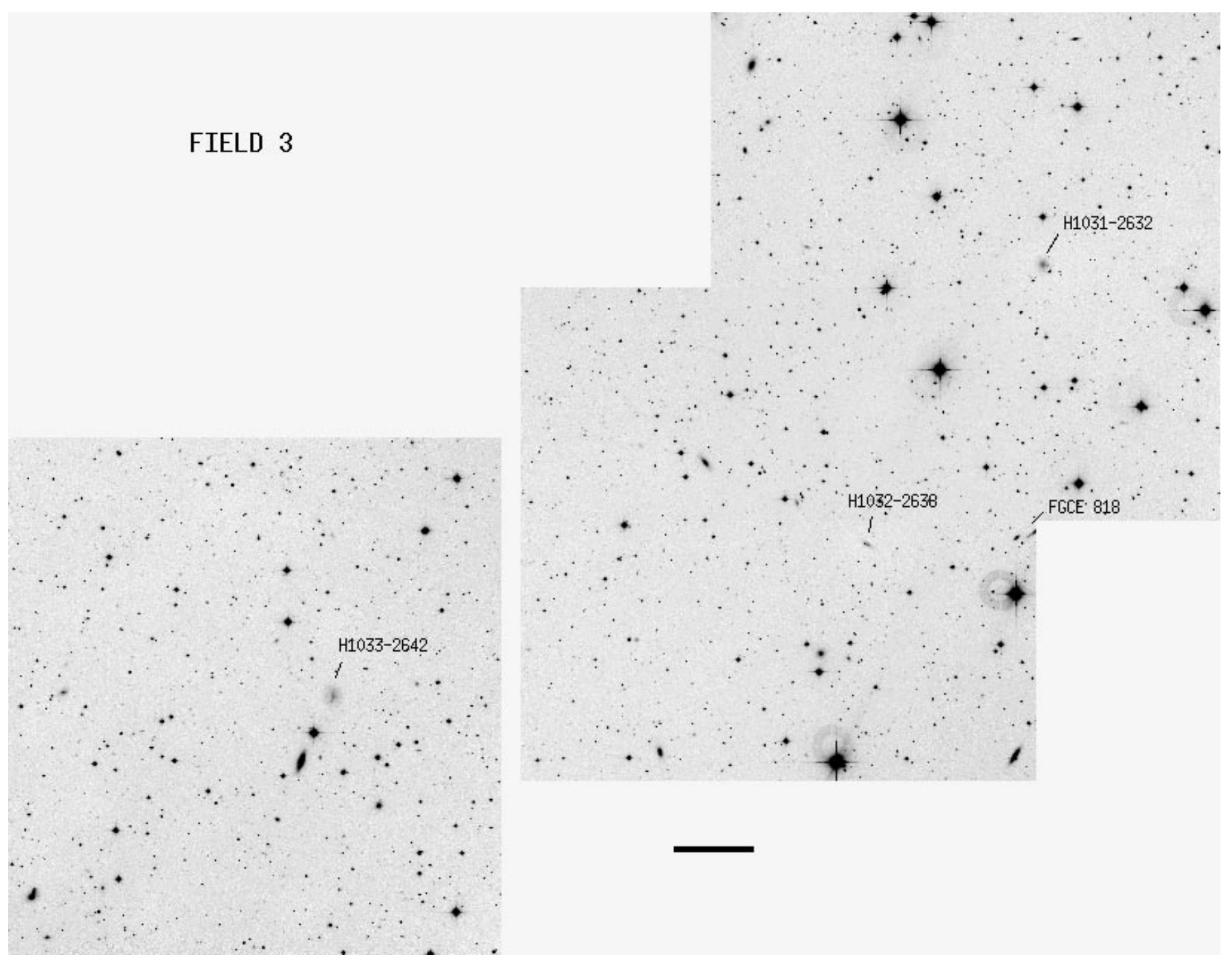

Fig. 4. Identification chart of Field 3 (see Fig. 2 for details)

via the ESO SKYCAT tool. The images were corrected for distortions during the same process. The seeing in the $I$-band varied between $1^{\prime \prime} 1$ and 1 ." 5 with a median value of 1 !. 4 .

\subsection{Near-infrared imaging}

Near-infrared images in the $K^{\prime}$ band were obtained in March 1998 with the IRAC2-B instrument on the ESO/MPI $2.2 \mathrm{~m}$ telescope using a $256 \times 256$ NICMOS3 array with a $2^{\prime} \cdot 1 \times 2.1$ field of view and a pixel size of 0 "'.5. The observations were made during full moon, causing strong reflections in the telescope that gave rise to important background variations in the frames which could not always be corrected for. The accuracy of the photometric measurements is largely limited by this problem, especially for low surface brightness objects. Different observing templates with various sets of offsets were used, with the aim of maximizing the exposure time on target. Sky images have either been extracted from the target images ("On" positions) or from "empty" adjacent fields ("Off" positions) depending on the object's shape and size. Typically, Off images had to be taken for objects more extended that $1 / 5^{\text {th }}$ of the detector field of view. The exposure time was basically adjusted according to the surface brightness of the galaxies. For various reasons, the actual total exposure times listed in Table 3 may depart from the initial estimates. They range between 5 and 60 minutes. The highest values were obtained in the favorable cases where the galaxy was small enough to be kept always on the detector. A total of 12 out of 15 galaxies from the final list were observed in the $K^{\prime}$ band.

Data reduction was carried out semi-automatically with IRAF scripts written by Duc and Lidman. All images were flat-fielded with an illumination-corrected dome flat and then sky-subtracted. Sky images were obtained by averaging dithered frames with a min-max rejection algorithm to get rid of infrared sources. The skysubtracted object images were first registered 


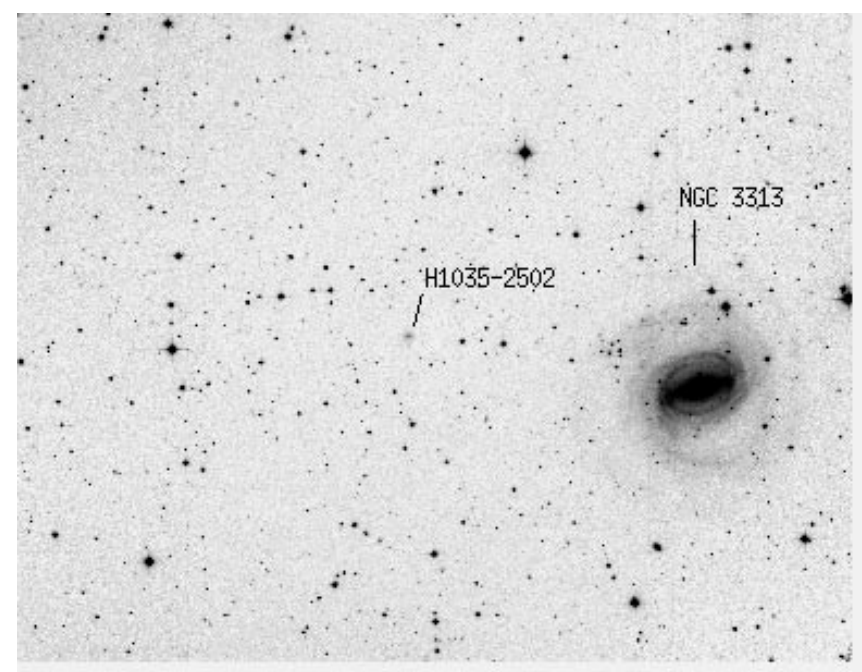

\section{FIELD 4}

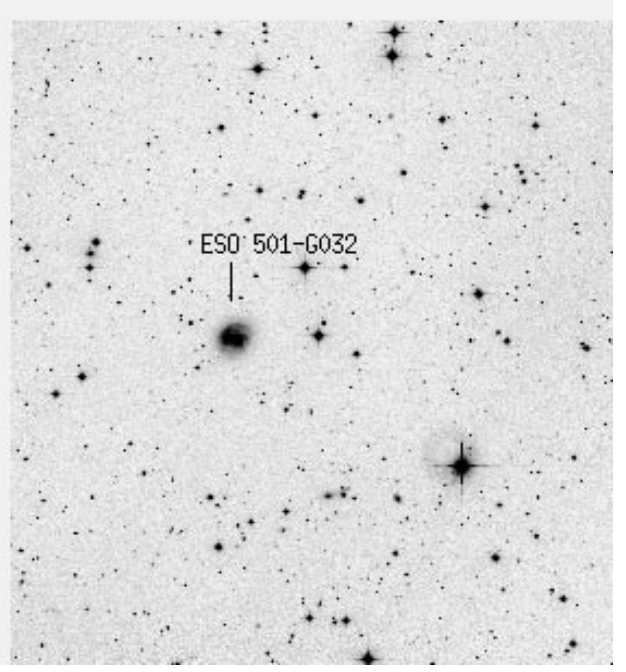

Fig. 5. Identification chart of Field 4 (see Fig. 2 for details)

FIELD 5
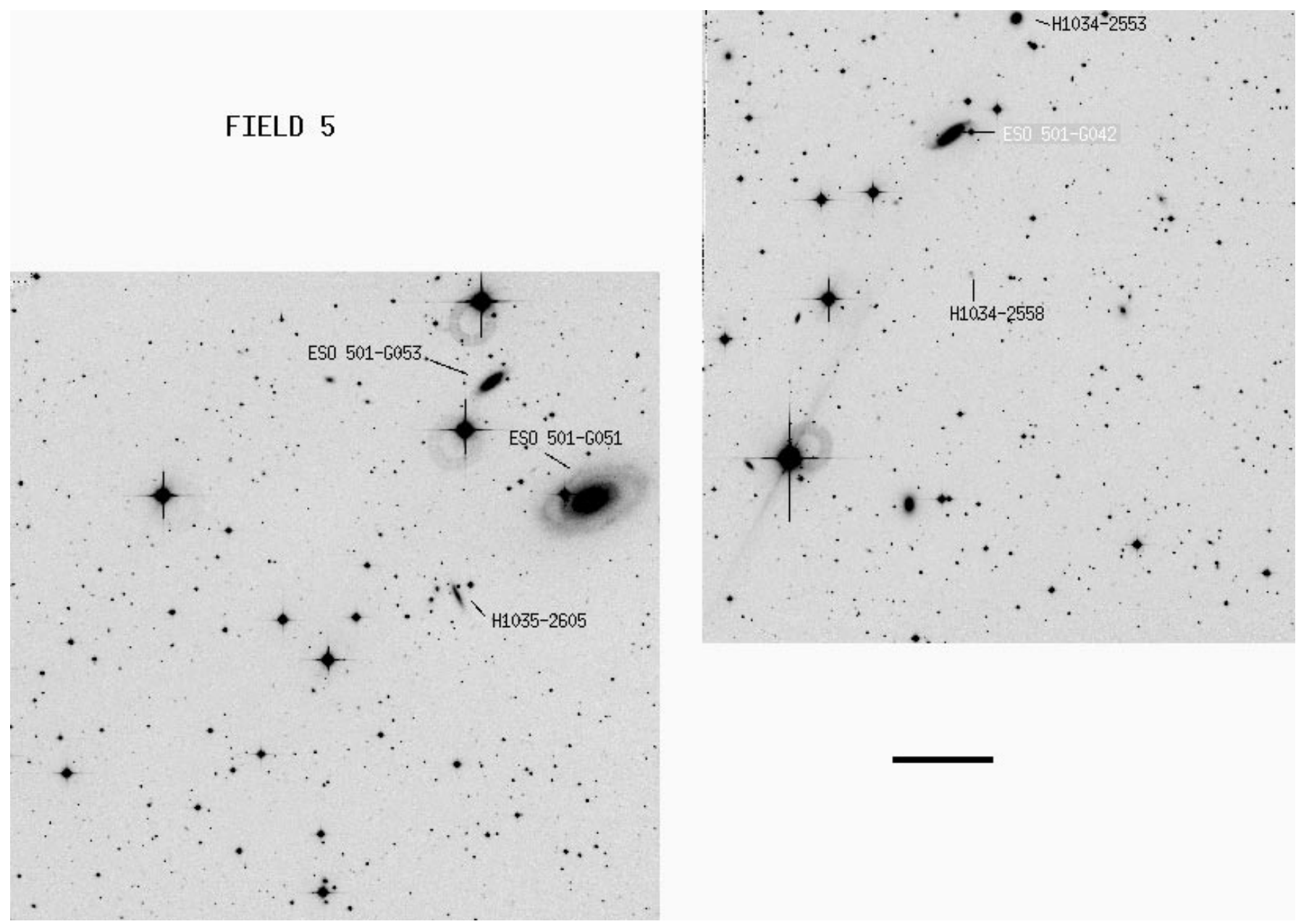

Fig. 6. Identification chart of Field 5 (see Fig. 2 for details) 


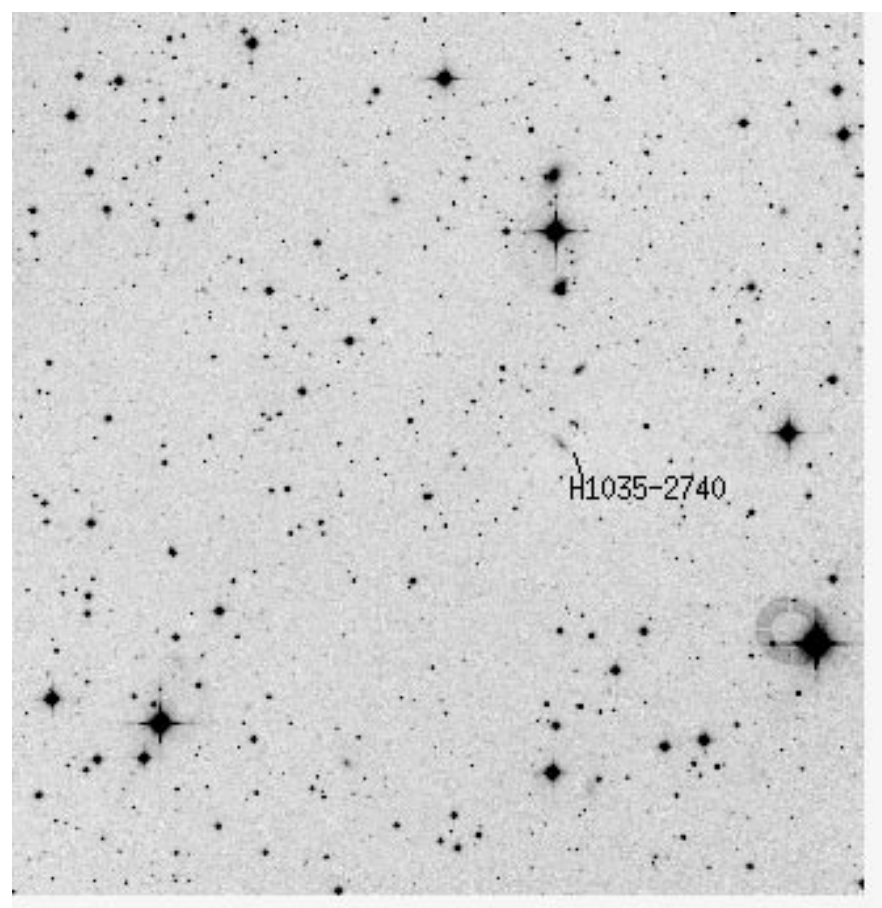

\section{FIELD 6}

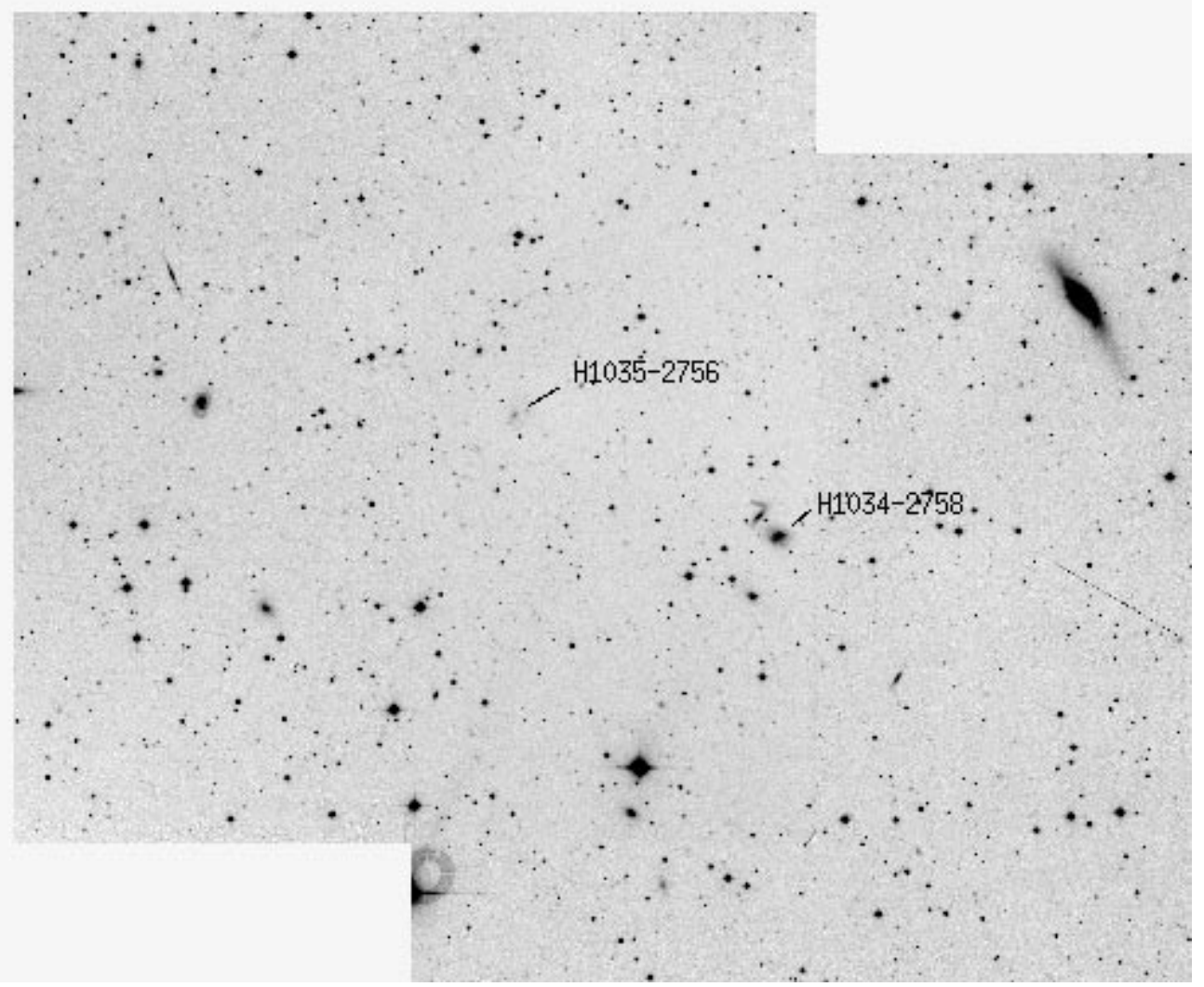

Fig. 7. Identification chart of Field 6 (see Fig. 2 for details) 


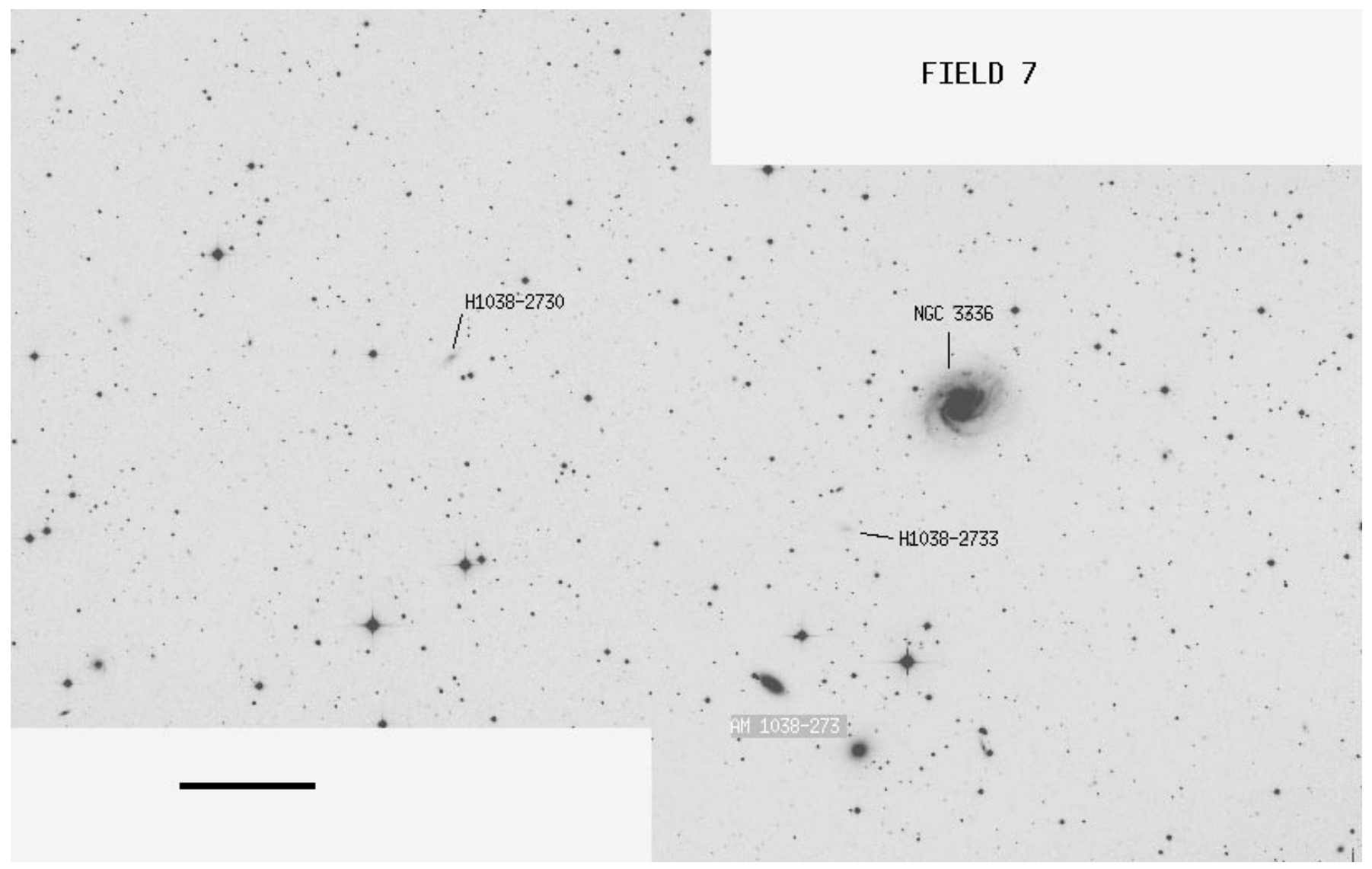

Fig. 8. Identification chart of Field 7 (see Fig. 2 for details)

automatically using the rough telescope offset information available as FITS header parameters and then more precisely using the positions of isolated common reference stars. We skipped all images showing large-scale, strong background variations, mainly due to reflections from the moon. The remaining frames were shifted and added with the task IMCOMBINE in IRAF, applying a sigma clipping rejection parameter.

Faint infrared standard stars from the NICMOS project (Persson et al. 1998) were used for photometric calibration. An extinction coefficient was fitted but no color corrections were applied. Weather conditions varied throughout the observing run from partly cloudy to photometric. They produced some systematic errors in the photometry that are difficult to estimate but that might be as high as $0.5 \mathrm{mag}$.

\section{Results}

\subsection{H I optical, and near-infrared detections and identifications}

The 16 brightest VLA H I sources of our primary list were re-observed in $\mathrm{HI}$ with the Nançay radio telescope. We confirm the presence of the $21 \mathrm{~cm}$ line at the position and velocity of all 16 VLA objects. The reduced Nançay spectra are shown in Fig. 9, after smoothing in velocity to a resolution of $15.6 \mathrm{~km} \mathrm{~s}^{-1}$. Global $\mathrm{H} \mathrm{I}$ line parameters were measured using standard Nançay data analysis software. The resulting central velocities, integrated line fluxes, line widths at $20 \%$ and $50 \%$ of peak maximum and the rms noise at the abovementioned velocity resolution are listed in Table 2. No corrections for instrumental resolution were applied to these values. The large telescope beam, HPBW 3'6 $\times 22^{\prime}(\alpha \times \delta)$, could in principle cause confusion with line signals from nearby $\mathrm{H}$ I-rich objects within the $1200 \mathrm{~km} \mathrm{~s}^{-1}$ bandwidth. In fact, a second emission line is seen in the spectra of 3 objects (H1031 - 2632, H1035 - 2502 and H1038 - 2733; see Fig. 9). However, in all cases the confusion problem could be solved and emission lines disentangled using the Nançay and original VLA imaging data in combination with optical radial velocities from the NED and LEDA databases (see Sect. 5). In general, the VLA and Nançay data agree quite well as far as the integrated line flux and the central profile velocity is concerned; a direct comparison of the line widths given in Table 2 is difficult, given the low velocity resolution of the VLA data and the fact that the widths quoted by McMahon (1993) are indicative only (see Sect. 2). 

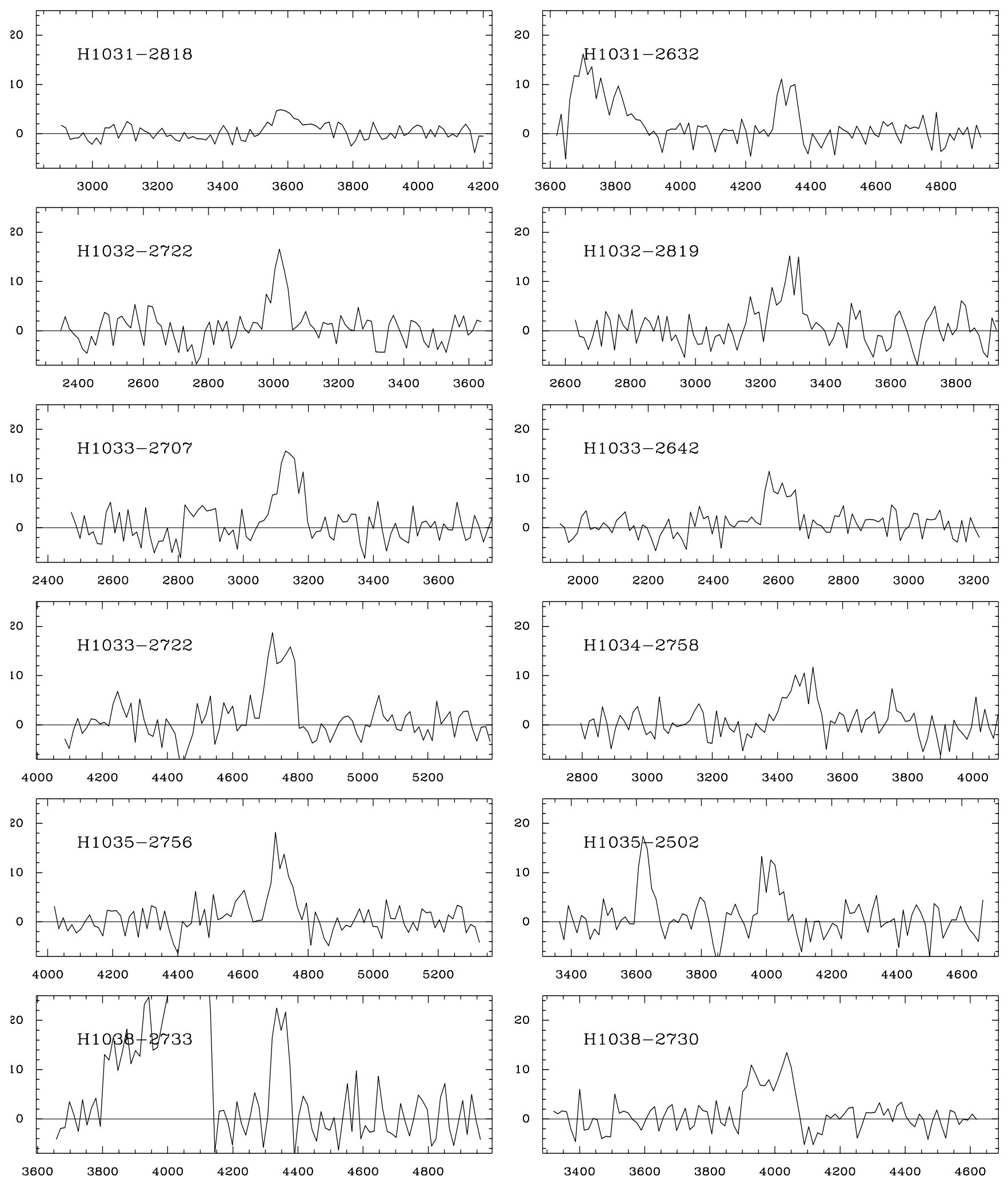

Fig. 9. $21 \mathrm{~cm} \mathrm{H}$ I line spectra of the $12 \mathrm{H} \mathrm{I-selected} \mathrm{dwarf} \mathrm{galaxies} \mathrm{in} \mathrm{the} \mathrm{Hydra} \mathrm{cluster} \mathrm{re-observed} \mathrm{at} \mathrm{Nançay.} \mathrm{The} x$-axis is the heliocentric velocity in $\mathrm{km} \mathrm{s}^{-1}$, in the optical convention, and the $y$-axis is the flux density in mJy. The velocity resolution of the spectra is $15.6 \mathrm{~km} \mathrm{~s}^{-1}$ 


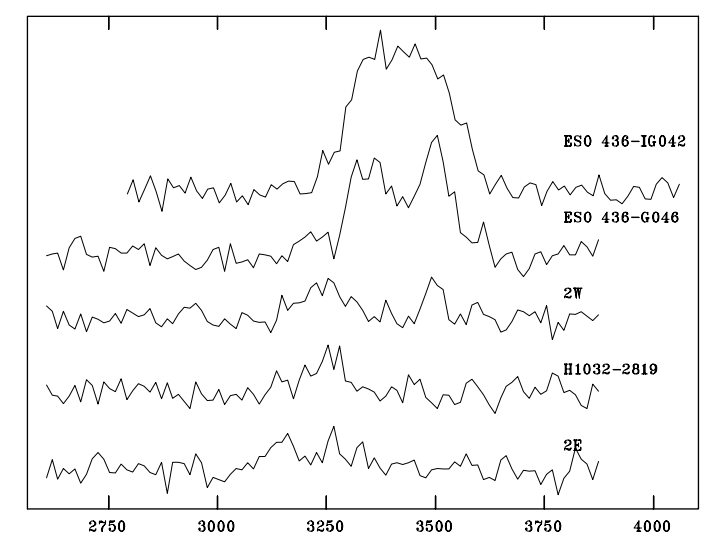

Fig. 10. Nançay H I spectra at various positions around H1032 - 2819, a VLA-H I source for which we found no clear optical counterpart. In the finding chart of the field (Fig. 3) we identified other sources in the vicinity that may in principle contribute to the emission line seen towards H1032 - 2819: ESO 436 - G046, 4.'3 to the West and ESO 436 - IG042 7'.2 to the West of H1032 - 2819. The optical centers of these two ESO galaxies were used as pointing centers for the Nançay H I spectra shown here; the spectra labeled $2 \mathrm{E}$ and $2 \mathrm{~W}$ were taken with pointing centers $2^{\prime}$ East and West, respectively, of the VLA H s source. Clearly the reality of the H I detection of H1032-2819 suffers no ambiguity
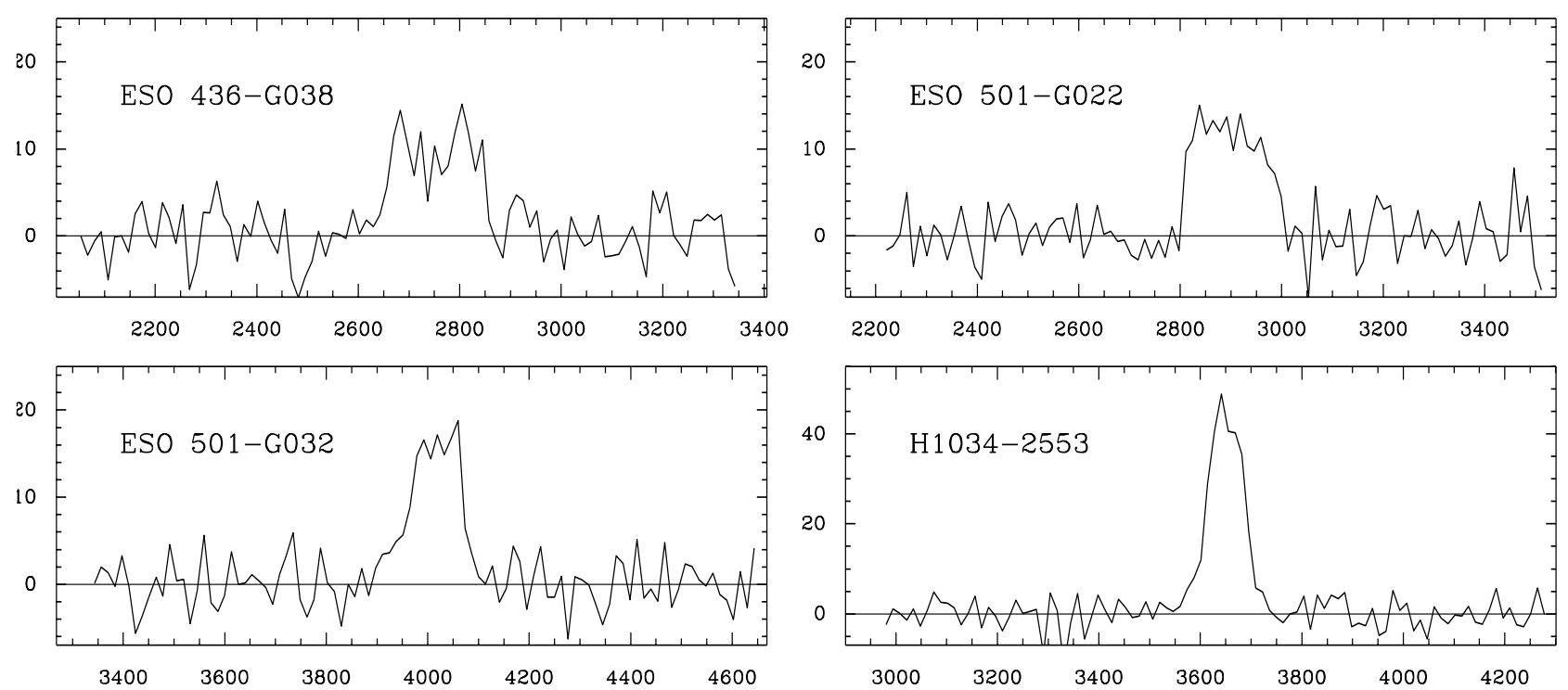

Fig. 11. Nançay H I spectra of 4 other galaxies that missed our dwarf galaxy selection criteria; see Fig. 9 for details

We found an optical counterpart to all but one of our 20 primary targets - at the location of the H I source H1032 - 2819 no extended stellar object was found, to an $I$-band surface brightness limit of $25 \mathrm{mag} \operatorname{arcsec}^{-2}$ (see Fig. 3). The closest optical object is a faint galaxy seen in the $I$-band $7^{\prime \prime}$ East of the central position of H1032 - 2819 and undetected in the $B$-band; it is most likely a red background galaxy. H1032 - 2819 is 4.3 and $134 \mathrm{~km} \mathrm{~s}^{-1}$ in radial velocity away from an $\mathrm{H} \mathrm{I-rich} \mathrm{spi-}$ ral galaxy, ESO 436 - G046. In the VLA data, this source is only $\mathrm{a} \sim 4 \sigma$ detection in a single channel map, but our Nançay observations pointed towards H1032 - 2819,
ESO 436 - G046 and two positions $2^{\prime}$ East and West of H1032 - 2819 (see Figs. 3 and 10) confirmed the reality of this H I source: it cannot be a sidelobe detection as its velocity does not match with that of any of the closeby sources. These observations will be discussed in a future Paper in these series.

Three candidate dwarf galaxies of the primary list appear on the CCD images as small face-on spirals: H1031 - 2734 (= ESO 436 - G038; Fig. 2), H1033 - 2506 (= the barred spiral ESO 501 - G032; Fig. 5) and H1034 - 2553 (included in the catalog of Richter et al. (1982), where it was misidentified as ESO 501 - G040, 


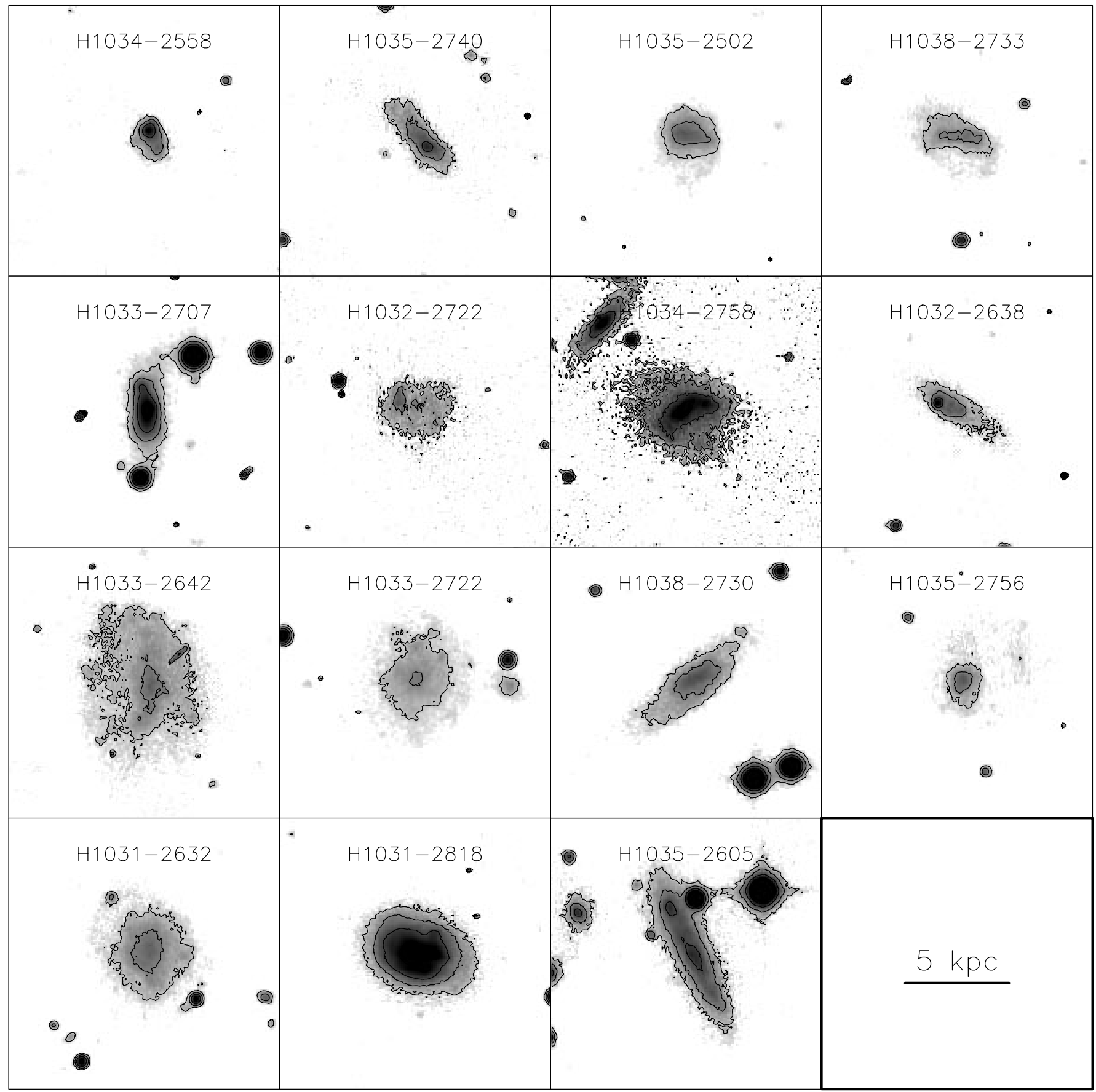

Fig. 12. $B$-band images of the $15 \mathrm{H}$ I-selected dwarf galaxies in the Hydra I cluster displayed with the same spatial and intensity scales. The galaxies are ordered according to their integrated $B-I$ color index, with the bluest objects displayed at the top left and the reddest at the bottom right. The lowest contour is $24.5 \mathrm{mag} \operatorname{arcsec}^{-2}$ and the interval is 0.5 mag. Note the diversity of the morphologies, ranging from compact objects to large low-surface brightness galaxies 


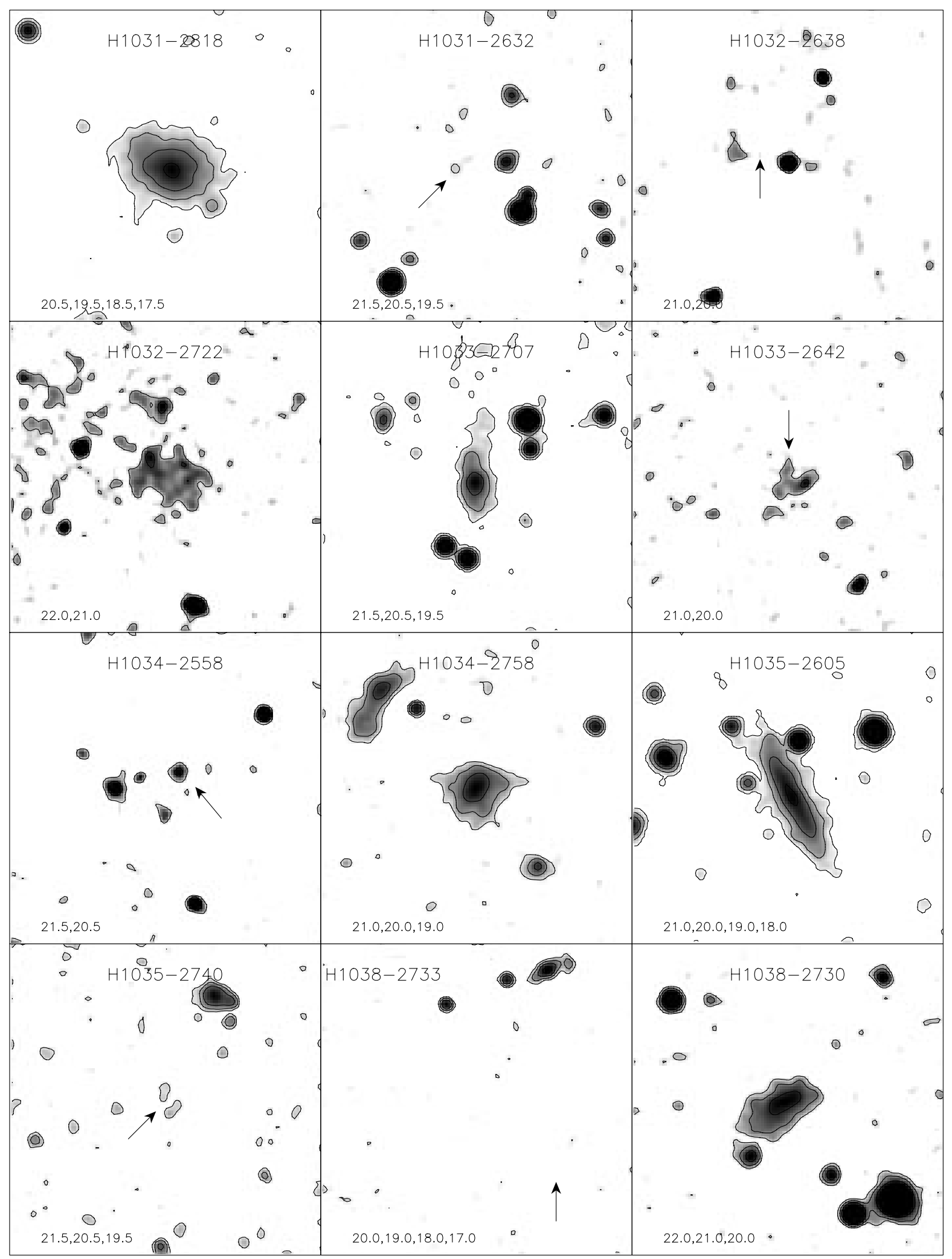

Fig. 13. $K^{\prime}$ - band images of the 12 dwarf galaxies observed at $2.2 \mu \mathrm{m}$ wavelength. The images have been smoothed with a circular Gaussian function and calibrated. The surface brightness levels of the overlayed contours in mag arcsec ${ }^{-2}$ are given at the bottom of each image. The arrows indicate the position of the dwarf galaxies in ambiguous cases. The size of each image is $1^{\prime} \times 1^{\prime}$. North is up and East left 

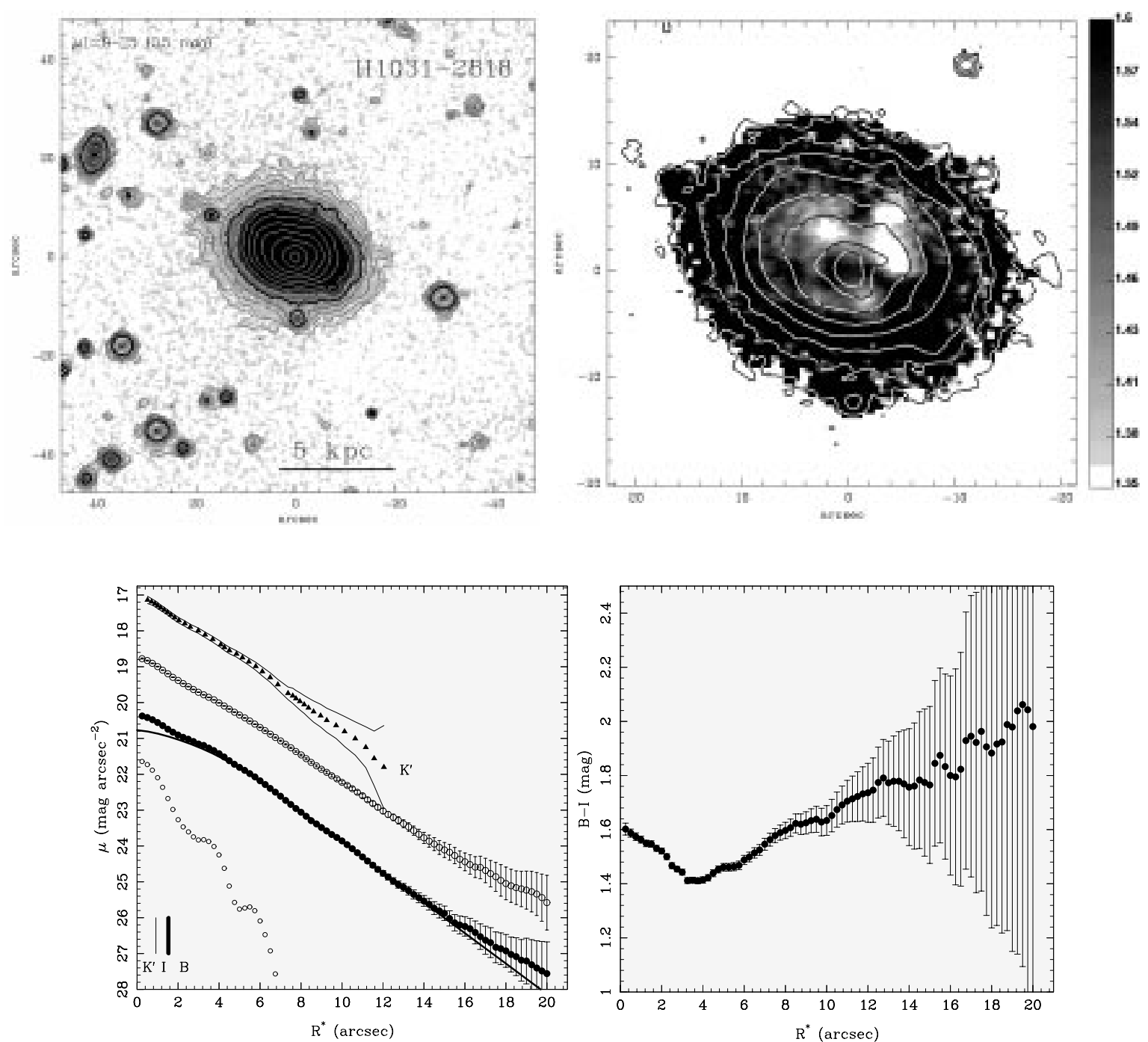

Fig. 14. H1031 - 2818. (top-left) I-band image. The thick contour shows the extent of the galaxy at the Galactic extinctioncorrected I surface brightness level of $23 \mathrm{mag} \operatorname{arcsec}^{-2}$. Contour levels are separated by 0.5 mag. (top-right): $B-I$ map of the galaxy. The overlayed contours correspond to the $B$-band image. (bottom-left) Surface brightness profiles in $B$ (filled circles), $I$ (open circles) and $K^{\prime}$ (triangles). The vertical error bars correspond to $2 \sigma$ uncertainties. The $F W H M$ of point sources is indicated by vertical lines of decreasing thickness for $B, I$ and $K^{\prime}$, respectively. The $B$-band SBP is decomposed into an LSB component (thick curve) plus the luminosity excess above the LSB profile (small circles). The luminosity of the LSB component can be described by a modified exponential distribution with $(b, q)=(1.8,0.65)$. (bottom-right) Radially averaged $B-I$ profile

however; see Fig. 5). Another galaxy with a more irregular morphology, H1033 - 2726 (ESO 501 - G022; Fig. 2), turns out to have an H I width (FWHM 168 $\mathrm{km} \mathrm{s}^{-1}$ ) considerably larger than the $130 \mathrm{~km} \mathrm{~s}^{-1}$ limit of our initial selection. The latter four objects have therefore all been excluded from further analysis. One galaxy, H1038 - 2730, with an H I line width $(F W H M$ $155 \mathrm{~km} \mathrm{~s}^{-1}$ ) exceeding slightly our selection criterion has nevertheless been kept in the sample as its absolute magnitude $\left(M_{B}=-15.8\right)$ lies clearly within the range of dwarf galaxies.

Among the 15 remaining dwarf galaxies, only 3 were already listed as catalogued elsewhere in the NED and LEDA databases (see Table 1): H1031 - 2818 as [R87]
103156.2 - 281833, PGC 31270 (Richter \& Huchtmeier 1987; Paturel et al. 1989), H1034 - 2558 as CTS 1015, M02.13 (Maza et al. 1991; Peña et al. 1991) and H1034 2758 as [R82] 103458.9-275843, PGC 31541 (Richter et al. 1982; Paturel et al. 1989). The calibrated images of all objects in our final list are shown in Figs. 14 to 28, and a thumbnail picture representation of all objects is displayed in Fig. 12. In the near-infrared $K^{\prime}$ band, 5 galaxies out of the 12 observed were detected clearly, 4 only marginally and 3 not at all (see Fig. 13). Limiting factors for detection were the central surface brightness of the galaxies as well as the stability of the sky background and, in general, weather conditions. 

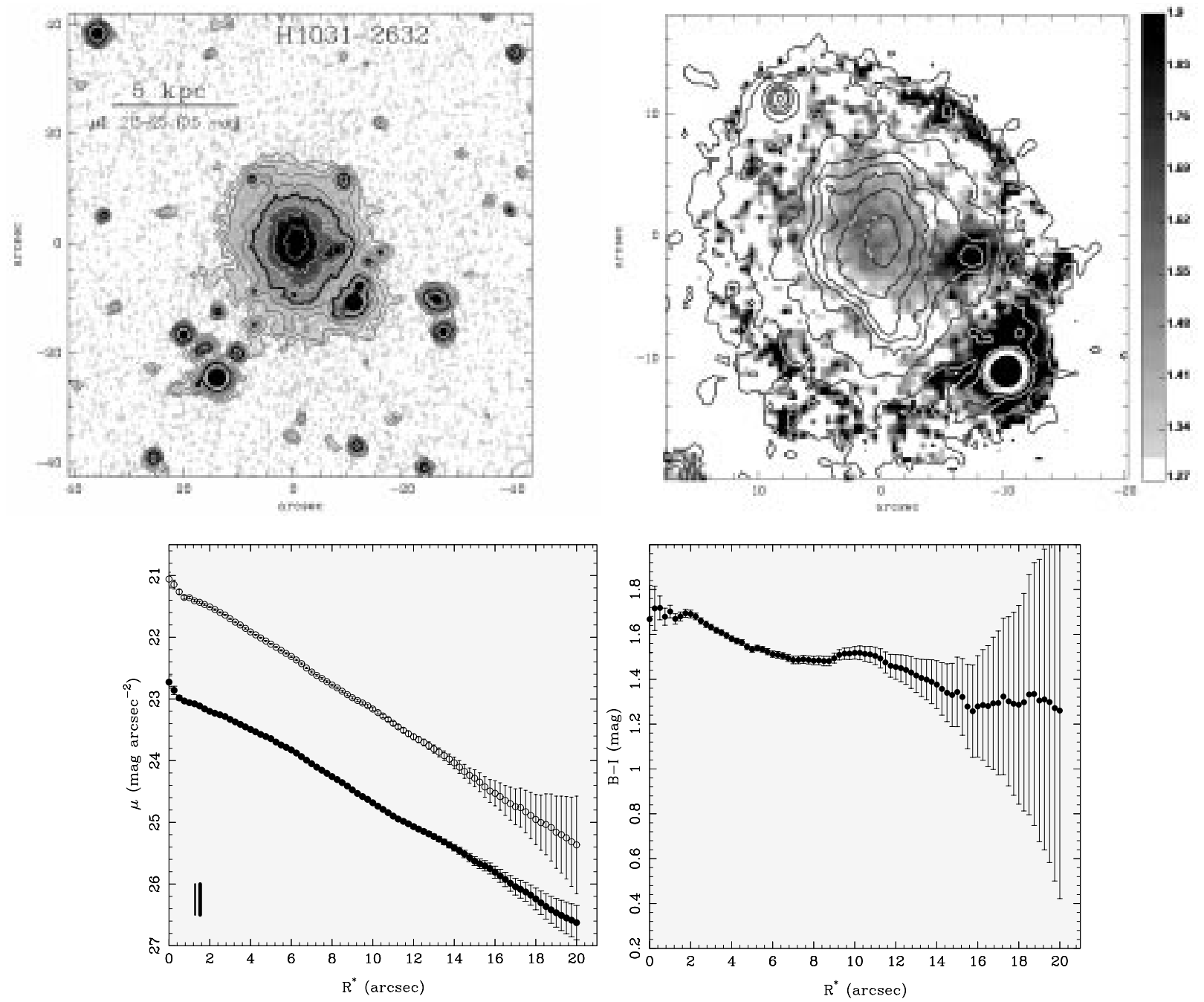

Fig. 15. H1031 - 2632. (top-left) I-band image. The thick contour shows the extent of the galaxy at the Galactic extinctioncorrected I surface brightness of $23 \mathrm{mag} \operatorname{arcsec}^{-2}$. Contour levels are separated by 0.5 mag. (top-right): $B-I$ map of the galaxy. The overlayed contours correspond to the $B$-band image. (bottom-left) Surface brightness profile in $B$ (filled circles) and $I$ (open circles), corrected for Galactic extinction. The vertical error bars correspond to $2 \sigma$ uncertainties. The $F W H M$ of point sources is indicated by the vertical thick and thin lines for $B$ and $I$, respectively. (bottom-right) Radially averaged $B-I$ profile

\subsection{Surface brightness profiles}

Surface brightness profiles (SBPs) were computed for the $B$ and $I$-bands following Papaderos et al. (1996ab), and displayed in Figs. 14 to 28 for the 15 dwarfs of our final list. The SBPs were corrected for foreground Galactic absorption. The extrapolated central surface brightness $\mu_{0}$ and exponential scale length $\alpha$ of the low surface brightness (LSB) disk component were derived from linear fits to the outer parts of each profile, weighted by the photometric uncertainties of each point. For three galaxies with sufficiently high surface brightness, an SBP could be derived in the $K^{\prime}$ band with the same method as used in the optical. It should be noted that the errors indicated for the $K^{\prime}$ profiles are purely statistical and do not take into account the systematic errors indicated above. Relevant photometric properties of the observed galaxy sample, corrected for foreground Galactic absorption, are listed in Table 4. Column 3 contains the extrapolated central surface brightness of the LSB disk component in units of mag $\operatorname{arcsec}^{-2}$ and Col. 4 the exponential disk scale length in pc. Columns 5 and 6 list the apparent luminosity and dimension of each dwarf determined at the surface brightness level of $25 \mathrm{mag} \operatorname{arcsec}^{-2}$. Column 7 contains the total apparent luminosity of each dwarf, as determined from a combination of numerical integration of each SBP out to the outermost point and the flux measurements within polygonal apertures, with contaminating foreground objects screened out. For $K^{\prime}$, the total magnitude, which could be determined for 5 objects only, has been measured within a polygonal aperture. Column 8 lists the effective radius for each profile in $\mathrm{kpc}$, and Col. 9 the concentration index CI, defined, after James (1991), as $\log \left(r_{80} / r_{20}\right)$, where $r_{80}$ and $r_{20}$ denote the radii within which $80 \%$ and 
Table 4. Photometric properties of the HI selected Hydra dwarfs

\begin{tabular}{|c|c|c|c|c|c|c|c|c|c|c|}
\hline Name & Band & $\begin{array}{c}\mu_{0} \\
\operatorname{mag} \square^{-2} \\
(3)\end{array}$ & $\begin{array}{c}\alpha \\
\mathrm{pc} \\
(4)\end{array}$ & $\begin{array}{c}m_{25} \\
\operatorname{mag} \\
(5)\end{array}$ & $\begin{array}{c}R_{25} \\
\mathrm{kpc} \\
(6)\end{array}$ & $\begin{array}{c}m_{\text {tot }} \\
\text { mag } \\
(7)\end{array}$ & $\begin{array}{c}R_{\text {eff }} \\
\mathrm{kpc} \\
(8) \\
\end{array}$ & 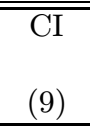 & $\begin{array}{c}\text { Seeing } \\
\operatorname{arcsec} \\
(10) \\
\end{array}$ & $\begin{array}{c}A_{B} \\
\text { fit range } \\
(11)\end{array}$ \\
\hline \multirow[t]{3}{*}{ H1031 - 2818} & $B$ & $19.64 \pm 0.02$ & $558 \pm 6$ & $15.81 \pm 0.02$ & 2.74 & $15.77 \pm 0.02$ & 1.05 & 0.503 & 1.54 & 0.17 \\
\hline & $I$ & $18.39 \pm 0.02$ & $618 \pm 5$ & $14.23 \pm 0.03$ & 3.89 & $14.20 \pm 0.03$ & 1.10 & 0.534 & 1." 47 & $>6^{\prime \prime}$ \\
\hline & $K^{\prime}$ & $16.54 \pm 0.34$ & $553 \pm 58$ & - & - & $12.69 \pm 0.1$ & 0.97 & 0.502 & $0 . .92$ & $>6^{\prime \prime}$ \\
\hline \multirow{2}{*}{ H1031 - 2632} & $B$ & $22.84 \pm 0.05$ & $1276 \pm 30$ & $17.52 \pm 0.012$ & 2.53 & $17.14 \pm 0.04$ & 1.80 & 0.496 & $1^{\prime \prime} 53$ & 0.2 \\
\hline & $I$ & $20.95 \pm 0.08$ & $1070 \pm 35$ & $15.68 \pm 0.05$ & 3.97 & $15.65 \pm 0.07$ & 1.67 & 0.511 & $1^{\prime \prime} 28$ & $>10^{\prime \prime}$ \\
\hline \multirow[t]{2}{*}{ H1032- 2638} & $\bar{B}$ & $22.29 \pm 0.05$ & $649 \pm 17$ & $18.26 \pm 0.04$ & 1.62 & $18.07 \pm 0.06$ & 0.94 & 0.515 & $1 . " 44$ & 0.2 \\
\hline & $I$ & $21.21 \pm 0.07$ & $672 \pm 25$ & $16.92 \pm 0.08$ & 2.30 & $16.90 \pm 0.08$ & 0.96 & 0.512 & $1^{\prime \prime} 21$ & $>4^{\prime \prime}$ \\
\hline \multirow{2}{*}{ H1032- 2722} & $B$ & $21.82 \pm 0.27$ & $706 \pm 60$ & $17.80 \pm 0.06$ & 2.06 & $17.57 \pm 0.11$ & 1.30 & 0.45 & $1 . .43$ & 0.18 \\
\hline & $I$ & $20.77 \pm 0.24$ & $715 \pm 54$ & $16.58 \pm 0.09$ & 2.80 & $16.55 \pm 0.10$ & 1.34 & 0.43 & $1^{\prime \prime} 12$ & $>8^{\prime \prime}$ \\
\hline \multirow[t]{3}{*}{ H1033- 2707} & $\bar{B}$ & $21.25 \pm 0.24$ & $528 \pm 39$ & $17.12 \pm 0.05$ & 1.85 & $17.06 \pm 0.06$ & 0.67 & 0.577 & $1^{\prime \prime} 91$ & 0.18 \\
\hline & $I$ & $20.15 \pm 0.28$ & $517 \pm 43$ & $16.10 \pm 0.07$ & 2.25 & $16.09 \pm 0.07$ & 0.69 & 0.576 & $1^{\prime \prime} 41$ & $>6^{\prime \prime}$ \\
\hline & $K^{\prime}$ & $18.56 \pm 0.18$ & $484 \pm 55$ & - & - & $15.02 \pm 0.15$ & 0.65 & 0.531 & 0.' 93 & $>2^{\prime \prime}$ \\
\hline \multirow[t]{2}{*}{ H1033- 2642} & $B$ & $22.99 \pm 0.03$ & $2184 \pm 73$ & $16.76 \pm 0.04$ & 3.63 & $16.56 \pm 0.06$ & 2.27 & 0.438 & $1^{\prime \prime} .49$ & 0.18 \\
\hline & $I$ & $21.56 \pm 0.05$ & $1873 \pm 88$ & $15.33 \pm 0.03$ & 4.50 & $15.31 \pm 0.09$ & 2.18 & 0.466 & 1.. 26 & $5^{\prime \prime}-14^{\prime \prime}$ \\
\hline \multirow[t]{2}{*}{ H1033- 2722} & $\bar{B}$ & $23.25 \pm 0.16$ & $1784 \pm 180$ & $17.44 \pm 0.04$ & 2.89 & $17.04 \pm 0.06$ & 2.21 & 0.476 & $1 . .89$ & 0.17 \\
\hline & $I$ & $21.70 \pm 0.22$ & $1458 \pm 162$ & $15.805 \pm 0.13$ & 4.35 & $15.77 \pm 0.18$ & 2.02 & 0.504 & 1.. 39 & $>10^{\prime \prime}$ \\
\hline \multirow[t]{2}{*}{ H1034-2558 } & $B$ & $22.28 \pm 0.18$ & $483 \pm 31$ & $18.33 \pm 0.02$ & 1.21 & $18.19 \pm 0.05$ & 0.56 & 0.63 & $1^{\prime \prime} 59$ & 0.26 \\
\hline & $I$ & $21.53 \pm 0.17$ & $526 \pm 36$ & $17.49 \pm 0.04$ & 1.70 & $17.43 \pm 0.04$ & 0.56 & 0.67 & 1.. 33 & $>5^{\prime \prime}$ \\
\hline \multirow[t]{3}{*}{ H1034-2758 } & $\bar{B}$ & $20.97 \pm 0.04$ & $897 \pm 17$ & $16.03 \pm 0.06$ & 3.15 & $\lesssim 16.02 \pm 0.06$ & 1.29 & 0.546 & $1 . .43$ & 0.16 \\
\hline & $I$ & $19.94 \pm 0.03$ & $912 \pm 13$ & $14.93 \pm 0.04$ & 4.25 & $\lesssim 14.92 \pm 0.04$ & 1.28 & 0.555 & $1^{\prime \prime} 34$ & $>5^{\prime \prime}$ \\
\hline & $K^{\prime}$ & - & - & - & - & $14.61 \pm 0.1$ & - & - & - & - \\
\hline \multirow[t]{2}{*}{ H1035-2756 } & $B$ & $23.86 \pm 0.10$ & $1724 \pm 178$ & $18.50 \pm 0.06$ & 1.80 & $17.72 \pm 0.18$ & 1.77 & 0.568 & $1^{\prime \prime} 58$ & 0.16 \\
\hline & $I$ & $22.49 \pm 0.14$ & $1645 \pm 240$ & $16.37 \pm 0.39$ & 2.82 & $16.305 \pm 0.24$ & 1.89 & 0.564 & 1." 43 & $>5^{\prime \prime}$ \\
\hline \multirow[t]{3}{*}{ H1035 - 2605} & $B$ & $21.50 \pm 0.12$ & $878 \pm 36$ & $16.62 \pm 0.034$ & 2.82 & $16.53 \pm 0.05$ & 1.34 & 0.477 & $1^{\prime \prime} 72$ & 0.26 \\
\hline & $I$ & $19.38 \pm 0.11$ & $738 \pm 24$ & $14.84 \pm 0.04$ & 3.83 & $14.84 \pm 0.04$ & 1.23 & 0.487 & $1^{\prime \prime} 35$ & $>9^{\prime \prime}$ \\
\hline & $K^{\prime}$ & $17.32 \pm 0.33$ & $618 \pm 56$ & — & 一 & $13.26 \pm 0.1$ & 0.87 & 0.490 & 1..06 & $>4^{\prime \prime} .4$ \\
\hline \multirow[t]{2}{*}{ H1035 - 2502} & $B$ & $22.17 \pm 0.05$ & $683 \pm 19$ & $18.05 \pm 0.04$ & 1.79 & $17.90 \pm 0.07$ & 1.00 & 0.480 & $1^{\prime \prime} 97$ & 0.22 \\
\hline & $I$ & $21.34 \pm 0.07$ & $728 \pm 32$ & $16.97 \pm 0.11$ & 2.29 & $16.97 \pm 0.11$ & 1.03 & 0.478 & $1^{\prime \prime} 50$ & $>4^{\prime \prime}$ \\
\hline \multirow[t]{2}{*}{$\mathrm{H} 1035-2740$} & $B$ & $21.75 \pm 1.34$ & $529 \pm 177$ & $18.01 \pm 0.04$ & 1.64 & $17.88 \pm 0.06$ & 0.87 & 0.497 & $1^{\prime \prime} 64$ & 0.16 \\
\hline & $I$ & $20.95 \pm 0.78$ & $592 \pm 144$ & $16.97 \pm 0.12$ & 2.13 & $16.96 \pm 0.13$ & 0.91 & 0.494 & 1.. 51 & $>8^{\prime \prime}$ \\
\hline \multirow[t]{2}{*}{ H1038 - 2733} & $B$ & $22.96 \pm 0.04$ & $986 \pm 25$ & $18.25 \pm 0.02$ & 1.87 & $17.93 \pm 0.04$ & 1.25 & 0.49 & $1 . .71$ & 0.16 \\
\hline & $I$ & $21.79 \pm 0.07$ & $870 \pm 32$ & $17.00 \pm 0.07$ & 2.59 & $16.97 \pm 0.08$ & 1.18 & 0.50 & $1^{\prime \prime} 45$ & $>5^{\prime \prime}$ \\
\hline \multirow[t]{3}{*}{ H1038 - 2730} & $\bar{B}$ & $22.26 \pm 0.03$ & $913 \pm 14$ & $17.53 \pm 0.03$ & 2.27 & $17.42 \pm 0.05$ & 1.25 & 0.477 & 1.446 & 0.16 \\
\hline & $I$ & $20.79 \pm 0.04$ & $845 \pm 21$ & $16.04 \pm 0.09$ & 3.35 & $16.04 \pm 0.09$ & 1.18 & 0.506 & 1." 40 & $4^{\prime \prime}$ \\
\hline & $K^{\prime}$ & - & - & - & - & $15.30 \pm 0.15$ & - & - & - & - \\
\hline
\end{tabular}

\section{Notes}

$\mu_{0}$ : Extrapolated central surface brightness of the LSB component in mag $\operatorname{arcsec}^{-2}$.

$\alpha$ : Exponential scale length of the LSB component in pc. A distance of $45 \mathrm{Mpc}$ was assumed throughout.

$m_{25}$ : Extinction-corrected apparent luminosity within the $25 \mathrm{mag} \operatorname{arcsec}^{-2}$ isophote.

$R_{25}$ : Radial extent at the $25 \mathrm{mag} \operatorname{arcsec}^{-2}$ isophotal level, in kpc.

$m_{\text {tot }}$ : Total extinction-corrected magnitude.

$R_{\text {eff }}$ : Effective radius in kpc.

CI: Concentration Index $\log \left(r_{80} / r_{20}\right)$, where $r_{80}$ and $r_{20}$ denote the radii containing $80 \%$ and $20 \%$ of the total object luminosity, respectively (after James 1991).

Seeing: the $F W H M$ of the stellar sources measured in the field of the galaxies.

$A_{B}$ : Assumed Galactic extinction in $B$.

Fit Range: Range in radius in which fits were performed. 

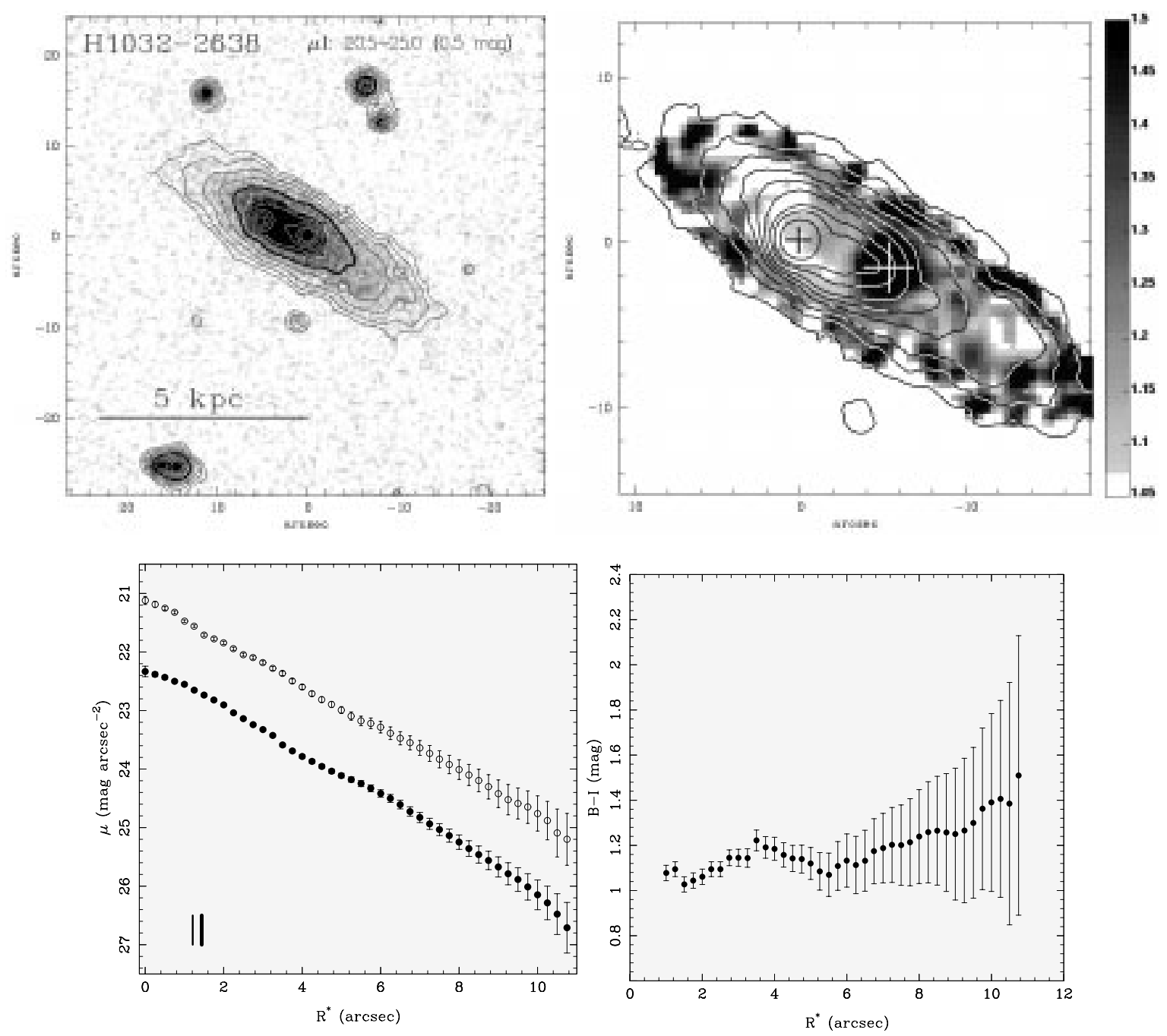

Fig. 16. H1032 - 2638. (top-left) I-band image. The thick contour shows the extent of the galaxy at the Galactic extinctioncorrected I surface brightness of $23 \mathrm{mag} \operatorname{arcsec}^{-2}$. Contour levels are separated by 0.5 mag. (top-right): $B-I$ map of the galaxy. The overlayed contours correspond to the $B$-band image. The crosses indicate the position of two spectroscopically identified foreground stars that were removed when computing the SBPs. (bottom-left) Surface brightness profile in $B$ (filled circles) and $I$ (open circles), corrected for Galactic extinction. The vertical error bars correspond to $2 \sigma$ uncertainties. The $F W H M$ of point sources is indicated by the vertical thick and thin lines for $B$ and $I$, respectively. (bottom-right) Radially averaged $B-I$ profile

$20 \%$ of the total luminosity are contained, respectively. In Col. 10 we list the seeing, i.e. the $F W H M$ (in arcsec) of stellar sources in the field of each dwarf. Column 11 contains the adopted Galactic extinction in the $B$-band and the range in radius used for the linear disk fit to each profile.

The LSB component of the SBPs can be divided in three main categories:

\section{$i$. Pure exponential profiles}

The surface brightness distribution of the LSB component of these dwarfs can be approximated, in at least one band, by a normal exponential law of the form:

$I\left(R^{*}\right)=I_{0} \exp \left(-\frac{R^{*}}{\alpha}\right)$ where $R^{*}$ is the radius, $I_{0}$ the central intensity and $\alpha$ the exponential disk scale length. About half the dwarfs in our sample belong to this category and show, within the $1 \sigma$ uncertainties, a profile shape compatible with Eq. (1). These objects are H1032 - 2638, H1033 - 2707, H1033 - 2722, H1034 - 2558, H1034 - 2758, H1035 - 2740, H1038 - 2733 and H1038 - 2730.

\section{ii. Exponential profiles flattening near the center}

These profiles, designated as type- $\mathrm{V}$ in the nomenclature of Binggeli \& Cameron (1991), exhibit an intensity depression with respect to a pure exponential distribution in their inner parts, typically for radii $\lesssim 2 \alpha$. In these cases the extrapolated central surface brightness of the LSB component, $\mu_{0}$, may differ significantly from the actually observed central surface brightness value. 

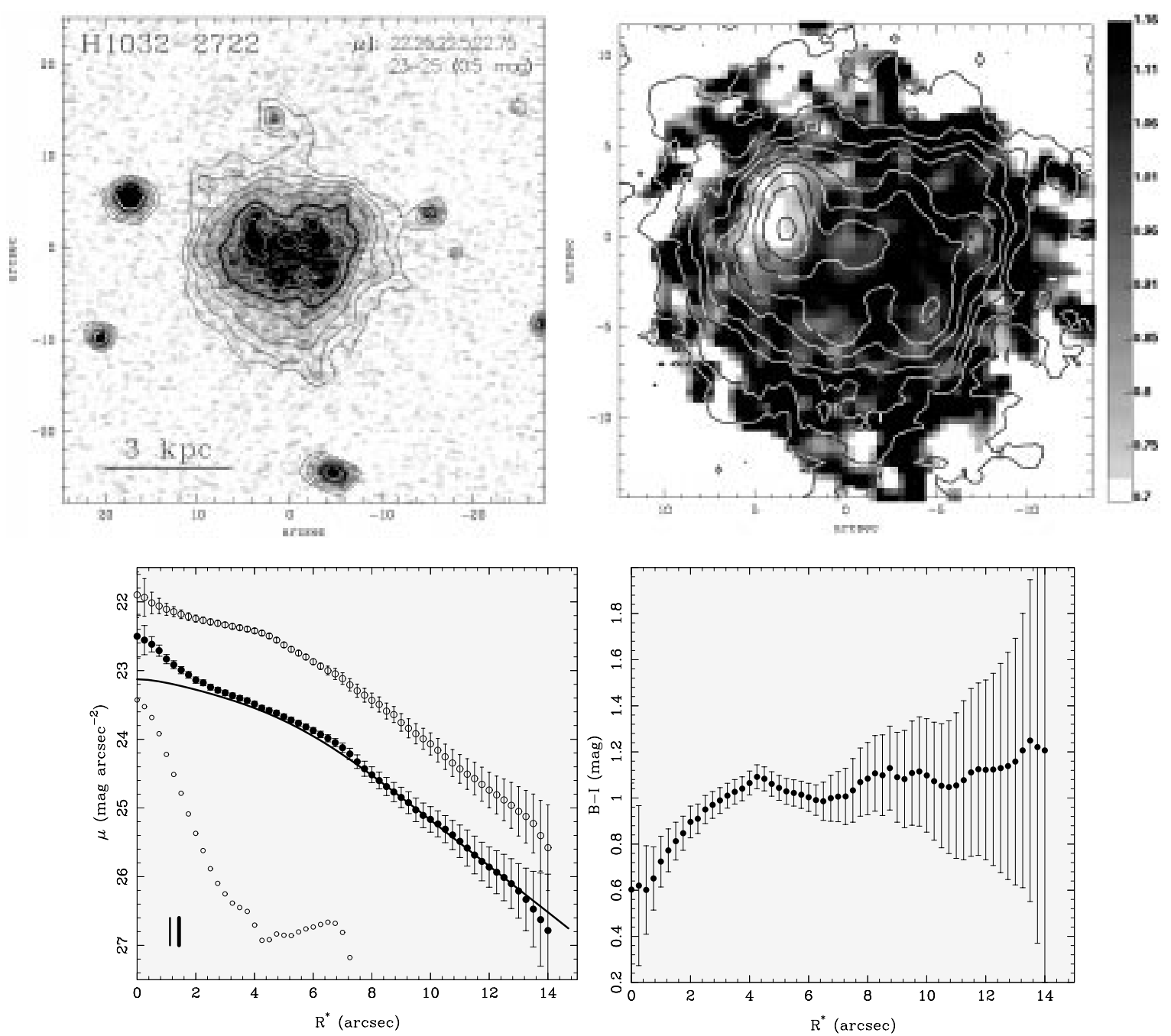

Fig. 17. H1032 - 2722. (top-left) $I$-band image. The thick contour shows the extent of the galaxy at the Galactic extinctioncorrected I surface brightness of $23 \mathrm{mag} \operatorname{arcsec}^{-2}$. Contour levels are separated by 0.5 mag. (top-right): $B-I$ map of the galaxy. The overlayed contours correspond to the $B$-band image. (bottom-left) Surface brightness profile in $B$ (filled circles) and $I$ (open circles), corrected for Galactic extinction. The vertical error bars correspond to $2 \sigma$ uncertainties. The $F W H M$ of point sources is indicated by the vertical thick and thin lines for $B$ and $I$, respectively. The $B$-band SBP is decomposed into an LSB component (thick curve) plus the luminosity excess above the LSB profile (small circles). The luminosity of the LSB component can be described by a modified exponential distribution with $(b, q)=(1.8,0.7)$. (bottom-right) Radially averaged $B-I$ profile

The LSB component of these galaxies can be described by a modified exponential distribution (Papaderos et al. $1996 \mathrm{~b})$ of the form

$I\left(R^{*}\right)=I_{0} \exp \left(-\frac{R^{*}}{\alpha}\right)\left\{1-q \exp \left(-P_{3}\left(R^{*}\right)\right)\right\}$

where $P_{3}\left(R^{*}\right)$ is defined as

$P_{3}\left(R^{*}\right)=\left(\frac{R^{*}}{b \alpha}\right)^{3}+\left(\frac{R^{*}}{\alpha} \frac{1-q}{q}\right)$.

The parameters $q=\Delta I / I_{0}$ and $b$ denote, respectively, the depression of the flattened exponential distribution with respect to a pure exponential distribution and the radial extent within which the flattening occurs.
Dwarfs with this type of profile constitute $\sim 1 / 3$ of our sample. They are H1031 - 2818, H1031 - 2632, H1032 2722, H1035 - 2605 and H1035 - 2502.

For two of these, we have illustrated the decomposition of the $B$-band profile using Eq. (2), as well as the residual light remaining after fitting. As shown in Figs. 14 and 17 , the $q$-parameter can be as high as 0.7 in the profile fits, implying an observed central surface brightness $\sim 1.3$ mag fainter than the extrapolated central value of the outer exponential profile. The physical origin of this type of profile is unclear; it has been conjectured that it may be due to early extensive mass loss via galactic winds (Fukunaga-Nakamura \& Tosa 1989).

iii. Peculiar profiles

These are observed in two cases: H1033-2642 and H1035- 

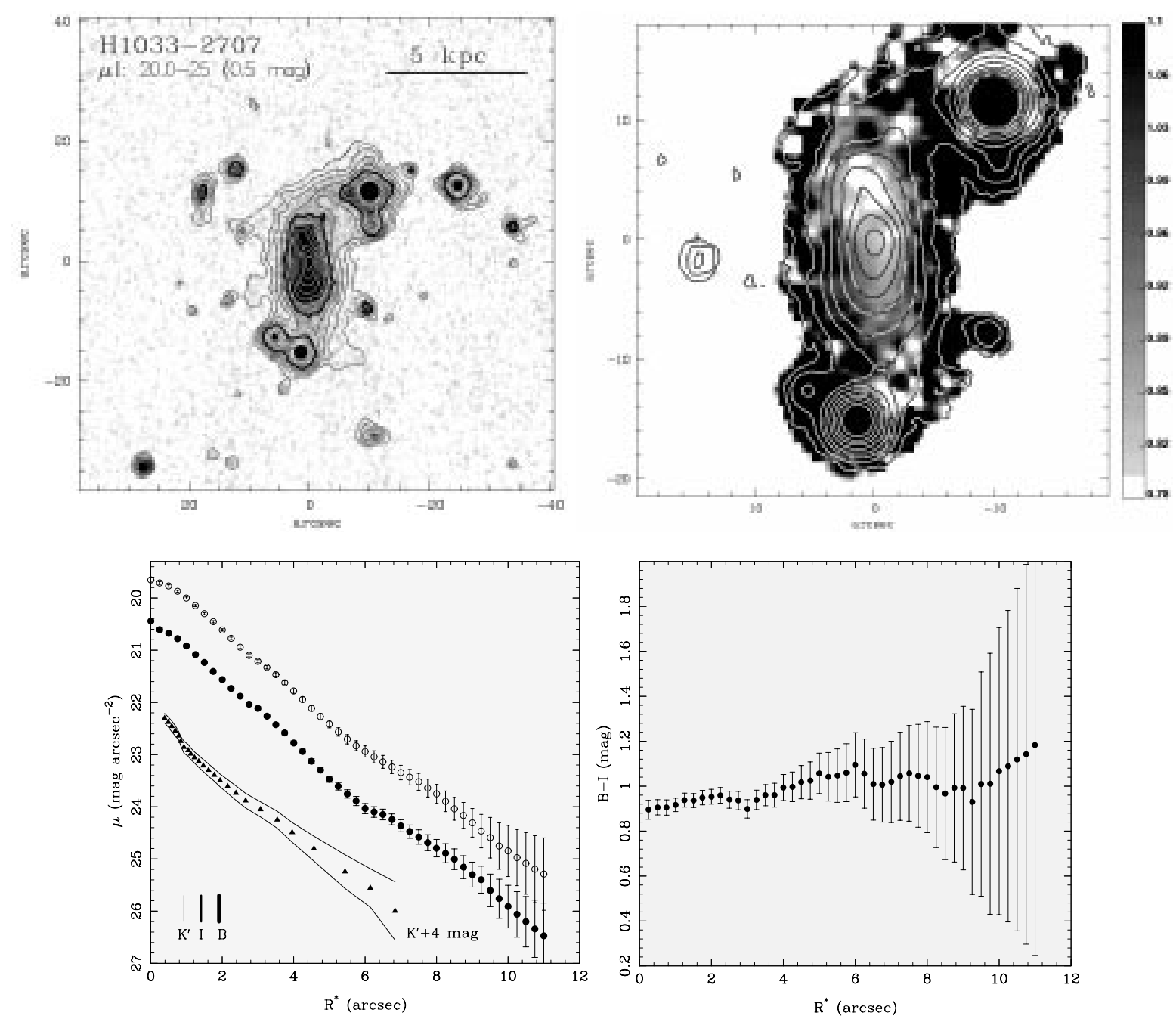

Fig. 18. H1033 - 2707. (top-left) I-band image. The thick contour shows the extent of the galaxy at the Galactic extinctioncorrected I surface brightness of $23 \mathrm{mag} \operatorname{arcsec}^{-2}$. Contour levels are separated by 0.5 mag. (top-right): $B-I$ map of the galaxy. The overlayed contours correspond to the $B$-band image. (bottom-left) Surface brightness profile in $B$ (filled circles), $I$ (open circles) and $K^{\prime}$ (triangles). An offset of $4 \mathrm{mag}$ has been added to the $K^{\prime}$ SBP. The vertical error bars correspond to $2 \sigma$ uncertainties. The $F W H M$ in each band is indicated at the bottom left. (bottom-right) Radially averaged $B-I$ profile

2756. The former shows an exponential intensity distribution for intensities brighter than $\mu_{B} \sim 24.5 \mathrm{mag} \operatorname{arcsec}^{-2}$ which is truncated in the outer regions. This truncation may be due to a tidal interaction with a nearby S0 galaxy. In H1035 - 2756 the LSB component appears filamentary and patchy, and it dominates the light for intensities fainter than $\mu_{B} \sim 25 \mathrm{mag} \operatorname{arcsec}^{-2}$.

\subsection{Color distribution}

The $B-I$ color maps are displayed at the upper right-hand panels of Figs. 14-28. The overlaying contours correspond to the $B$-band images. Note, that the color distribution of the dwarfs is not always spatially correlated with the $B$-band luminosity pattern; e.g. the intensity maximum does not necessarily coincide with a region exhibiting extreme colors with respect to the mean color of the galaxy.
In most cases the bluest colors are found off-center, at the location of spectroscopically confirmed H II regions (see Paper II). Examples of dwarfs with off-center color minima are H1031 - 2818, H1033 - 2722, H1034 - 2758, H1035 - 2605, H1035 - 2502 and H1038 - 2733.

Radial color profiles (Figs. 14-28, lower-right panels) were computed by direct subtraction of the $I$-band SBP from the $B$-band SBP, after matching both profiles to the same resolution. In general, no strong color gradients are observed in the sample. The only exception is H1034 - 2558, a blue compact dwarf galaxy showing a inward bluing by $1.8 \mathrm{mag} \mathrm{kpc}^{-1}$ within its starburst nucleus. The LSB components show a variety of radially averaged color distributions, ranging between weak outward bluing of the order of $\lesssim-0.2 \mathrm{mag} \mathrm{kpc}^{-1}$ and zero color gradient. 

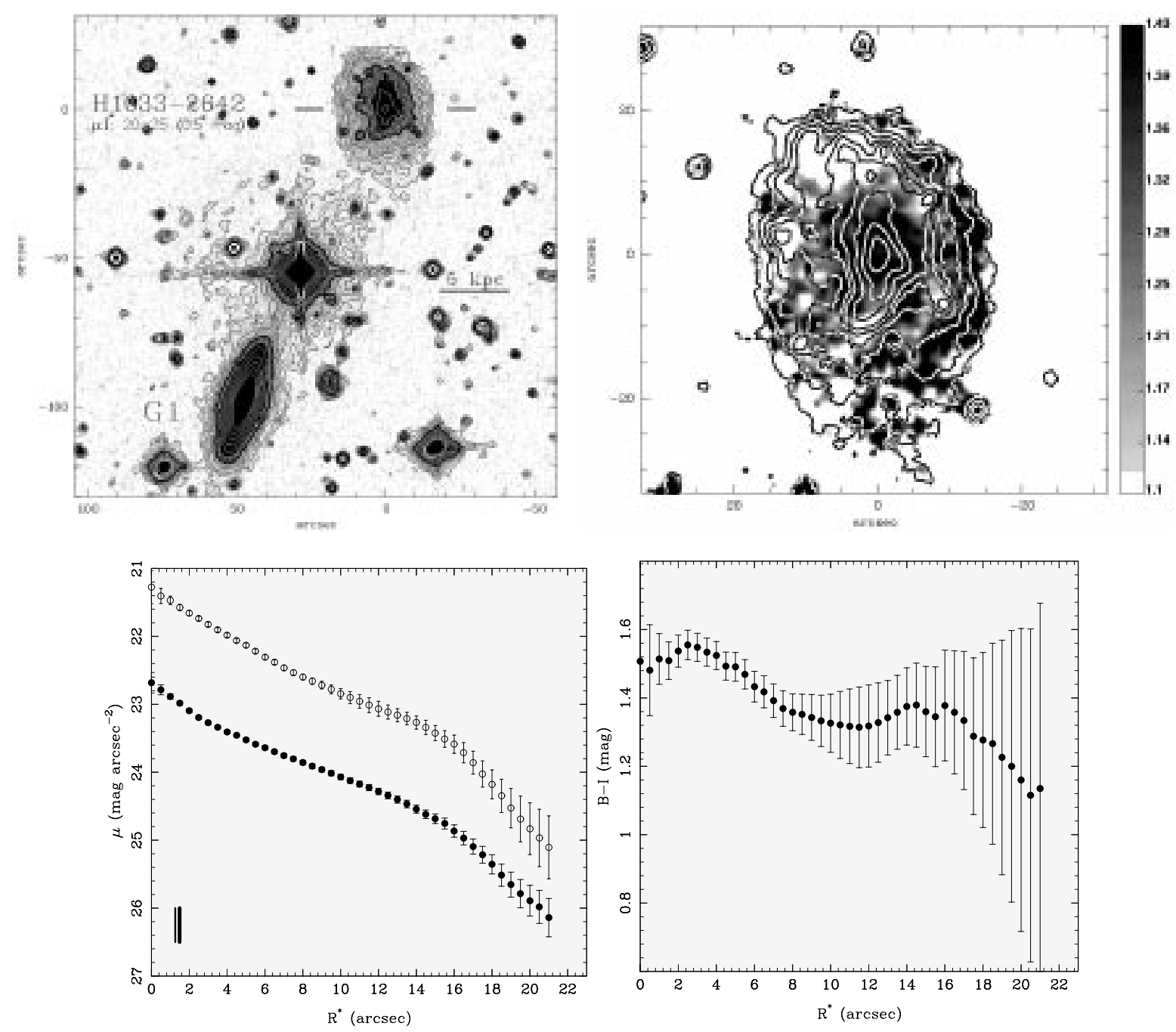

Fig. 19. H1033 - 2642. (top-left) I-band image. The thick contour shows the extent of the galaxy at the Galactic extinctioncorrected I surface brightness of $23 \mathrm{mag} \operatorname{arcsec}^{-2}$. Contour levels are separated by 0.5 mag. (top-right): $B-I$ map of the galaxy. The overlayed contours correspond to the $B$-band image. (bottom-left) Surface brightness profile in $B$ (filled circles) and $I$ (open circles), corrected for Galactic extinction. The vertical error bars correspond to $2 \sigma$ uncertainties. The $F W H M$ of point sources is indicated by the vertical thick and thin lines for $B$ and $I$, respectively. (bottom-right) Radially averaged $B-I$ profile

\subsection{Classification of the Hydra dwarfs}

Table 5 summarizes some integrated properties of the primary list objects. The absolute blue magnitude (Col. 2) and the $B-I$ color index (Col. 5) were calculated using the total magnitudes listed in Table 4. The H I masses (Col. 3) were derived from the Nançay data, when available; otherwise, the original VLA line fluxes were used (those values are listed in parentheses in Table 5). All galaxies in our final list of $\mathrm{HI}$-selected dwarf galaxies are fainter than absolute blue magnitude -17.5 and have $\mathrm{H}$ I masses ranging from $0.710^{8} M_{\odot}$ to $7.310^{8} M_{\odot}$. The mean H I-massto-blue luminosity ratios of the 15 Hydra dwarf galaxies in our final list is about $1 M_{\odot} / L_{\odot, B}$, a rather typical value for gas-rich dwarf irregulars (Thuan 1985; Skillman 1996; Hoffman et al. 1996), though for individual objects it ranges from a low 0.1 (for H1031-2818 and H1035-2605), a value rather befitting quiescent lenticulars, to values exceeding 2 (for H1035 - 2756 and H1038 - 2733) which are not unexceptional for dwarf irregulars, however.

From the morphological point of view, the dwarfs of the $\mathrm{H}$ I-selected sample show a striking variety of properties. This diversity is clearly illustrated in Fig. 12, where surface brightness images of all 15 dwarfs are displayed together with the same spatial and intensity scales. In Figs. 29a,b we compare, respectively, the $M_{B}$ vs. $\mu_{0}$ and the $M_{B}$ vs. $\log (\alpha)$ correlation of our sample galaxies with those of other dwarf irregulars (dIrrs) and blue compact dwarfs (BCDs) taken from various other samples (see Patterson \& Thuan 1996; Papaderos et al. 1996a, and references therein). In both diagrams the Hydra dwarfs span a wide range in the $\mu_{0}$ and $\log (\alpha)$ parameter space, from BCD-like objects to LSB dIrrs. Objects BCD H1034-2558 and LSB H1033 - 2642 are the two most extreme cases. 

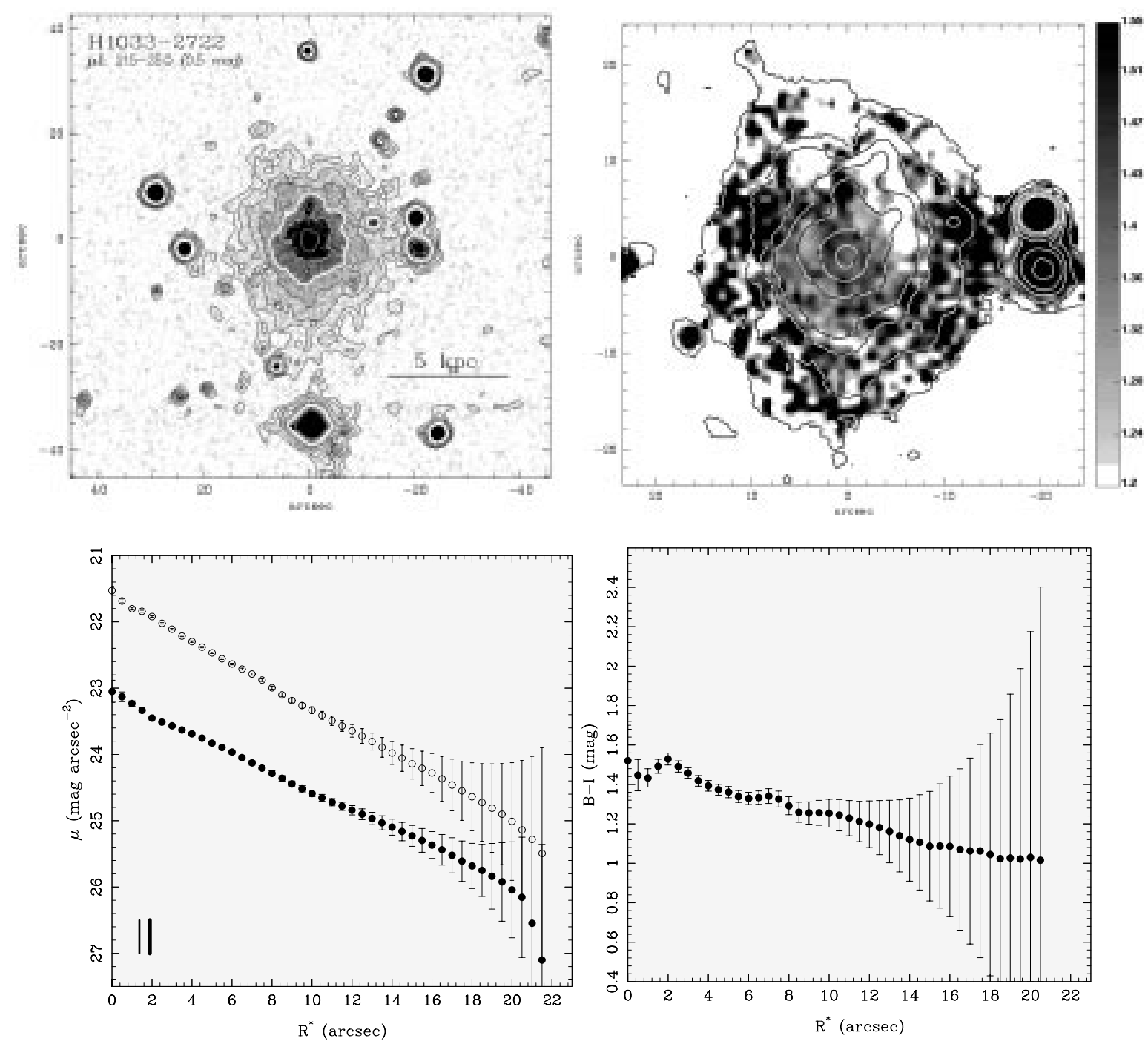

Fig. 20. H1033 - 2722. (top-left) I-band image. The thick contour shows the extent of the galaxy at the Galactic extinctioncorrected I surface brightness of $23 \mathrm{mag} \operatorname{arcsec}^{-2}$. Contour levels are separated by 0.5 mag. (top-right): $B-I$ map of the galaxy. The overlayed contours correspond to the $B$-band image. (bottom-left) Surface brightness profile in $B$ (filled circles) and $I$ (open circles), corrected for Galactic extinction. The vertical error bars correspond to $2 \sigma$ uncertainties. The $F W H M$ of point sources is indicated by the vertical thick and thin lines for $B$ and $I$, respectively. (bottom-right) Radially averaged $B-I$ profile

\section{Notes on individual objects}

We hereafter present some notes on each dwarf galaxy in the final list. The optical velocities of companion galaxies and the location of some $\mathrm{H}$ II regions mentioned were derived from long-slit spectroscopic data obtained in March 1998 with the ESO $3.6 \mathrm{~m}$ telescope. These observations will be presented in detail in Paper II.

- H1031 - 2818: This system is the most luminous dwarf in our sample, at $M_{B} \approx-17.5 \mathrm{mag}$. Its surface brightness profiles in both $B$ and $I$ suggest a central depression with respect to a normal exponential fitting law. This flattening appears more pronounced in $B$, where the extrapolation of the outer slope of the LSB component to $R^{*}=0^{\prime \prime}$ leads to a central surface brightness $\sim 0.7 B$ mag brighter than the observed value. In Fig. 14 the intensity distribution of the LSB component in $B$ is fitted with a modified exponential distribution of the form Eq. (2) with $(b, q)=(1.8,0.65)$. We determined the apparent $B$ luminosity of the LSB component as $15.83 \mathrm{mag}$ and that of the component in excess of it as 18.79 mag. Thus, the latter luminosity component, due to ongoing star-forming activity, represents only a minor contribution, $\sim 6 \%$, to the total luminosity of the system.

Within an area $\sim 14^{\prime \prime}$ in diameter there are three moderately blue $(B-I \sim 1.3 \mathrm{mag})$ off-center regions immersed in the relatively red $(B-I \sim 1.8)$ LSB stellar continuum. The latter knots host $\mathrm{H}$ II regions. For radii $\geq 4^{\prime \prime}$ the radially averaged $B-I$ profile shows a monotonic color increase with a gradient of $\sim 0.18 \mathrm{mag} \mathrm{kpc}^{-1}$. 

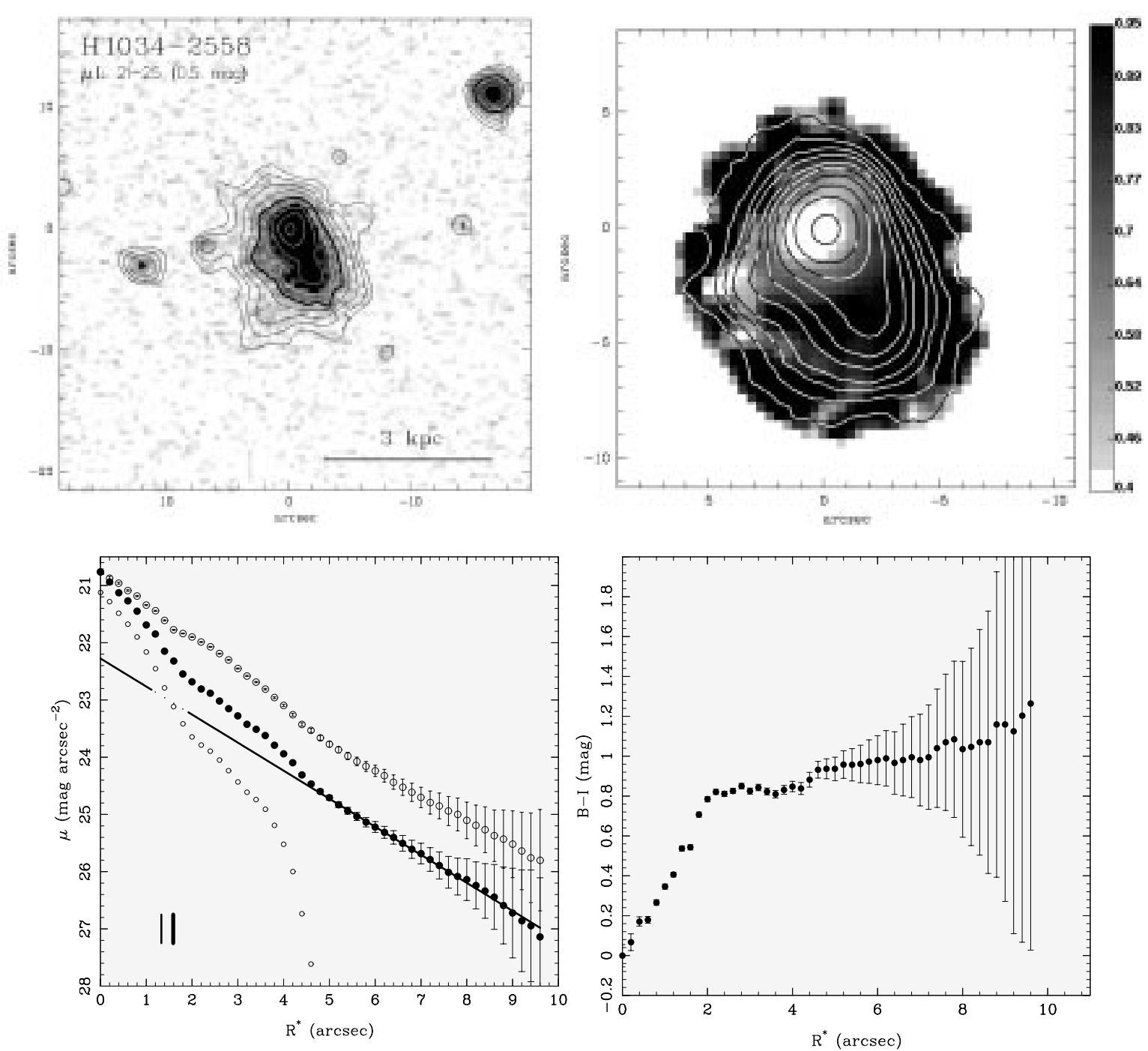

Fig. 21. H1034 - 2558. (top-left) $I$-band image. The thick contour shows the extent of the galaxy at the Galactic extinctioncorrected I surface brightness of $23 \mathrm{mag} \operatorname{arcsec}^{-2}$. Contour levels are separated by 0.5 mag. (top-right): $B-I$ map of the galaxy. The overlayed contours correspond to the $B$-band image. (bottom-left) Surface brightness profile in $B$ (filled circles) and $I$ (open circles), corrected for Galactic extinction. The vertical error bars correspond to $2 \sigma$ uncertainties. A decomposition into a pure exponential LSB component and a starburst component in excess of the LSB component is shown by the solid line and the small circles, respectively. The $F W H M$ of point sources is indicated by the vertical thick and thin lines for $B$ and $I$, respectively. (bottom-right) Radially averaged $B-I$ profile

This HI source is the only VLA HI source re-observed at Nançay but not reconfirmed. The upper limit to the integrated $\mathrm{H}$ I line flux listed in Table 2 is a $3 \sigma$ upper limit for an assumed line $F W H M$ of $75 \mathrm{~km} \mathrm{~s}^{-1}$, the average observed for the 15 confirmed primary list dwarf galaxies.

- H1031 - 2632: A low surface brightness object with a central blue surface brightness of only $23 \mathrm{mag} \operatorname{arcsec}^{-2}$. The galaxy is quite red $(B-I=1.5)$; it does not show any significant color gradient $\left(\Delta(B-I)=-0.11 \mathrm{mag} \mathrm{kpc}{ }^{-1}\right)$, nor evidence for star formation. The clump located roughly $8^{\prime \prime}$ West of the center $(B-I \sim 2)$ is presumably a background galaxy, which was also detected in $K^{\prime}\left(m_{K}=17.3\right)$. It was removed for the computation of surface brightness profiles. The Nançay H I spectrum shows a second signal, centered on $V_{\text {hel }}=3745 \mathrm{~km} \mathrm{~s}^{-1}$ with a $F W H M$ of $155 \mathrm{~km} \mathrm{~s}^{-1}$. In the NED and LEDA databases 2 small, highly inclined galaxies were found in an area 1.5 times the size of the telescope HPBW: FGCE 818, an 0'.9 diameter superthin Scd-type edge-on system (see Fig. 4) and ESO 501 - G017, an S0 at a redshift of $4429 \mathrm{~km} \mathrm{~s}^{-1}$. FGCE 818 is the obvious candidate for this H I signal, as shown by the VLA imaging of McMahon (1993) who measured $V_{\text {hel }}=3743 \mathrm{~km} \mathrm{~s}^{-1}$ for the VLA source.

H1032 - 2638: Edge-on dwarf galaxy. Two foreground stars, a blue and a red one, indicated in the color map (Fig. 16; top-right) by crosses, were removed when computing SBPs (though there may still remain some small 

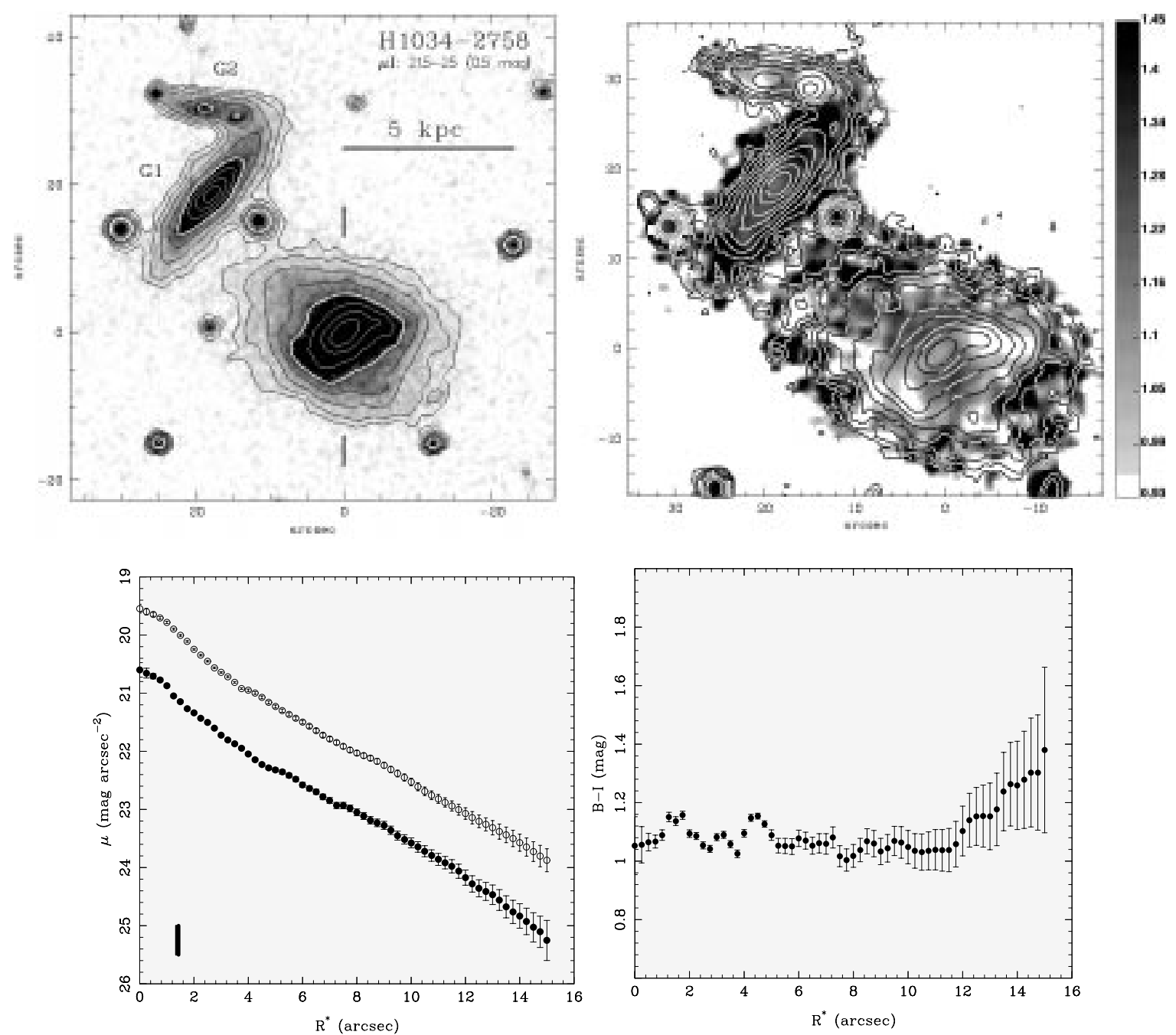

Fig. 22. H1034 - 2758. (top-left) I-band image. The thick contour shows the extent of each galaxy at the Galactic extinctioncorrected I surface brightness of $23 \mathrm{mag} \operatorname{arcsec}^{-2}$. Contour levels are separated by $0.5 \mathrm{mag}$. The H I-dwarf is indicated by the vertical thick lines. (top-right): $B-I$ map of the galaxy. The overlayed contours correspond to the $B$-band image. (bottom-left) Surface brightness profile in $B$ (filled circles) and $I$ (open circles), corrected for Galactic extinction. The vertical error bars correspond to $2 \sigma$ uncertainties. The $F W H M$ of point sources is indicated by the vertical thick and thin lines for $B$ and $I$, respectively. (bottom-right) Radially averaged $B-I$ profile, showing a nearly constant color

residuals). The galaxy itself was not detected in $K^{\prime}$. The blue region to the South-West hosts a strong H II region.

- H1032 - 2722: A low surface brightness object exhibiting an off-center blue clump. Its surface brightness profiles in both $B$ and $I$ suggest for radii $\leq 7^{\prime \prime}$ a central depression with respect to a normal exponential fitting law. In Fig. 17 the intensity distribution of the LSB component in $B$ is fitted with a modified exponential distribution of the form Eq. (2) with $(b, q)=(1.8,0.7)$.

The central surface brightness of the modified exponential distribution $\left(23.13 \mathrm{~B} \mathrm{mag} \operatorname{arcsec}^{-2}\right)$ is $1.3 \mathrm{mag}$ fainter than the extrapolated value of the outer slope $\left(21.82 \mathrm{mag} \operatorname{arcsec}^{-2}\right)$. The apparent $B$ luminosity of the LSB component was determined as $17.64 \mathrm{mag}$ and that of the excess light as $20.62 \mathrm{mag}$ (representing $\sim 6 \%$ of the total $B$ luminosity of the system). This H I-dwarf exhibits an off-center blue clump to the East. The latter shows up in $K^{\prime}$ whereas the low-surface brightness disk is barely detected in this band. Within the inner $1 \mathrm{kpc}$ $\left(\leq 4^{\prime \prime}\right)$ the radially averaged color profile shows a strong color gradient $\left(0.57 \mathrm{mag} \mathrm{kpc}^{-1}\right)$ whereas a constant blue color $(B-I=1.09 \pm 0.08)$ is measured in the LSB component. Widespread star formation is detected through spectroscopy. It is more active at the location of the blue clump.

H1032-2819: As discussed in Sect. 4.1, no optical counterpart was found to this H I cloud, situated less than $60 \mathrm{kpc}$ away from the spiral galaxy ESO 436 - G046 (see Fig. 3). One cannot exclude that this cloud has been detached from the spiral by tidal interaction. Although the latter does not exhibit strong signs of interaction, it has a nearby 

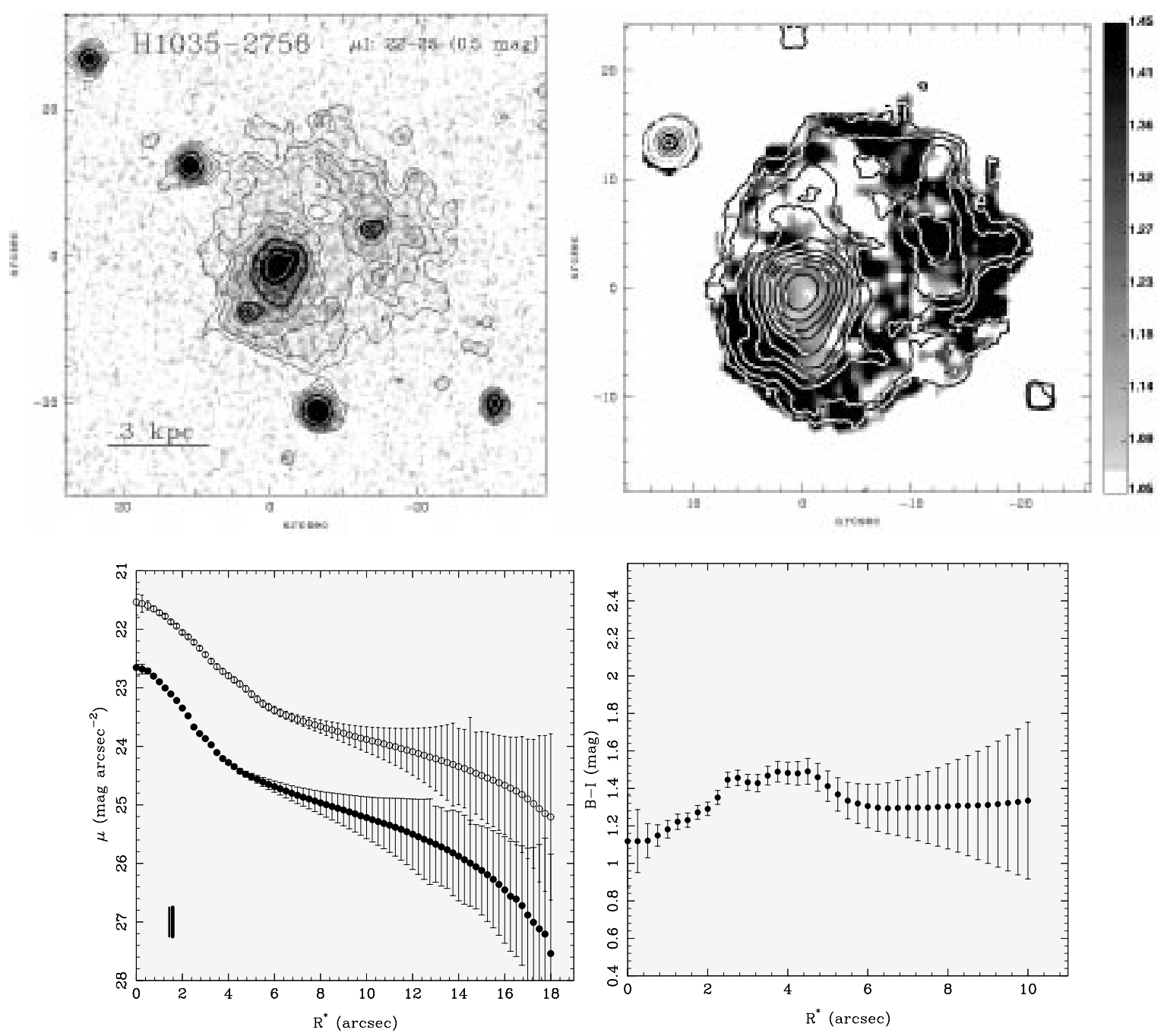

Fig. 23. H1035 - 2756. (top-left) I-band image. The thick contour shows the extent of the galaxy at the Galactic extinctioncorrected I surface brightness of $23 \mathrm{mag} \operatorname{arcsec}^{-2}$. Contour levels are separated by 0.5 mag. (top-right): $B-I$ map of the galaxy. The overlayed contours correspond to the $B$-band image. (bottom-left) Surface brightness profile in $B$ (filled circles) and $I$ (open circles), corrected for Galactic extinction. The vertical error bars correspond to $2 \sigma$ uncertainties. The $F W H M$ of point sources is indicated by the vertical thick and thin lines for $B$ and $I$, respectively. (bottom-right) Radially averaged $B-I$ profile

companion, ESO 436 - IG042 whose Nançay H I spectra is shown in Fig. 10.

- H1033 - 2707: A rather blue, high-surface brightness object, with an integrated $B-I$ index of $\sim 1.0$ mag. Colors are getting even bluer to the North along its major axis, where they attain a value $\sim 0.75$; a star forming region is observed there.

- H1033 - 2642: A red low-surface brightness object showing a truncation of its exponential profile at levels $\mu_{B} \gtrsim$ $25 \mathrm{mag} \operatorname{arcsec}^{-2}$. The galaxy is interacting with the S0 galaxy to the South-East (labeled as G1 in Fig. 19) which is at the same optical redshift $\left(V_{\mathrm{opt}}=2510 \mathrm{~km} \mathrm{~s}^{-1} ; m_{B}=\right.$ $\left.15.2 ; m_{I}=13.2\right)$. H1033 - 2642 has a red $(B-I \sim 1.45$ mag) central region with a $B-I$ index getting bluer $\left(-0.09 \pm 0.015 \mathrm{mag} \mathrm{kpc}{ }^{-1}\right)$ with increasing galactocen- tric radius. It is barely detected in $K^{\prime}$. A low level of starformation is seen all along the South-North axis.

H1033 - 2722: A red low-surface brightness object with a decreasing $B-I$ color towards larger radii $(-0.145 \pm$ $0.015 \mathrm{mag} \mathrm{kpc}-1)$. An off-centered blue region associated with an $\mathrm{H}$ II region is seen to the North-West.

- H1034 - 2558: A blue compact dwarf galaxy detected previously in the objective-prism survey by Maza et al. (1991) and catalogued as CTS 1015 and M01.13. It shows an extended, blue off-centered nucleus $(B-I \lesssim 0.2 \mathrm{mag})$ and a relatively constant color $(\sim 0.9-1.0 \mathrm{mag})$ in the LSB component. The averaged color gradient for radii $\leq 0.5$ $\mathrm{kpc}$ is as strong as $\sim 1.76 \mathrm{mag} \mathrm{kpc}-1$. The luminosity component in excess $\left(m_{B}=19.31 \mathrm{mag}\right)$ of the LSB component as modelled by a pure exponential law $\left(m_{B}=18.61\right)$ contributes $\lesssim 40 \%$ of the total light of H1034 - 2558, contrary to other $\mathrm{HI}$-detected dwarfs in our sample, which 

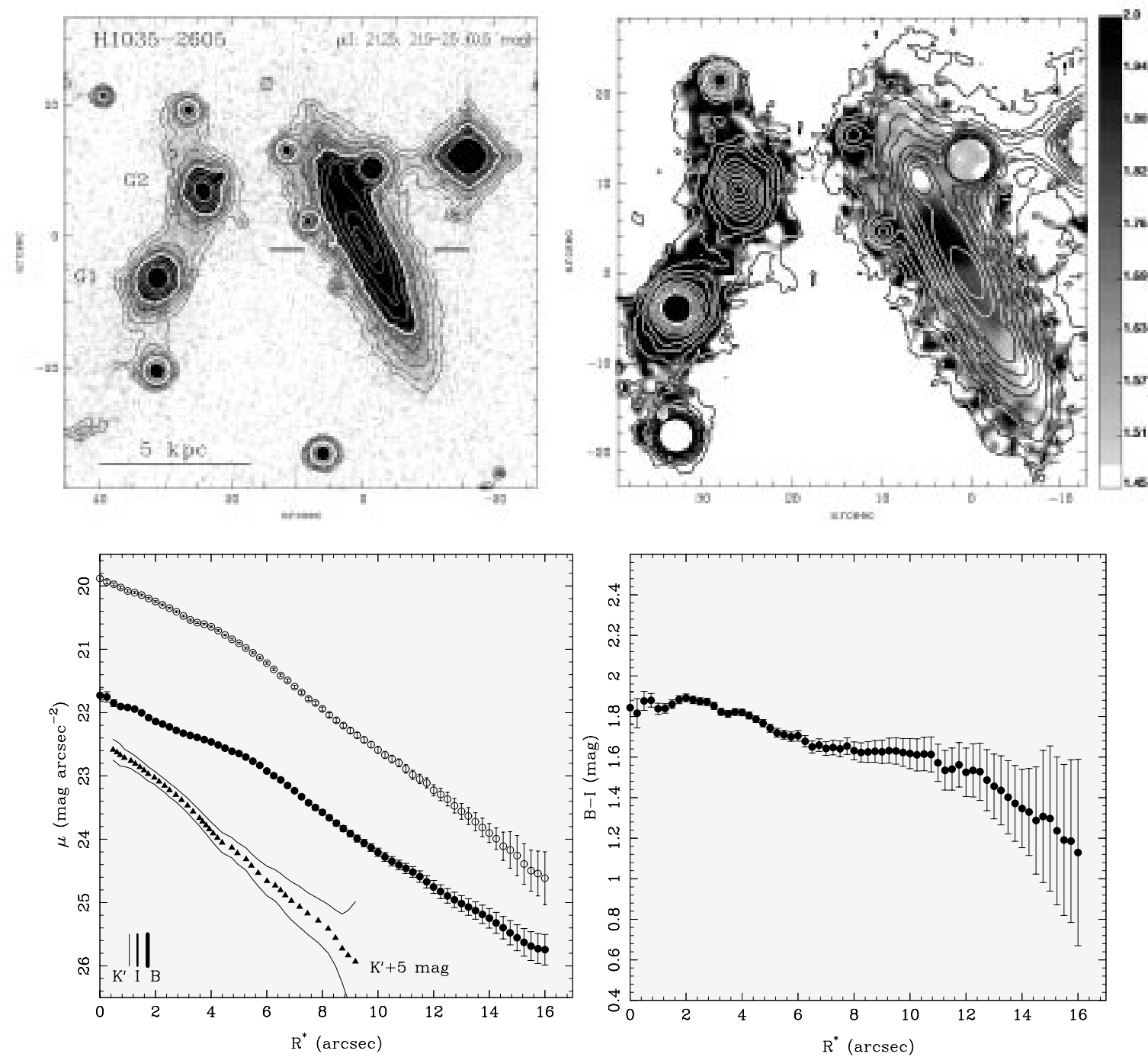

Fig. 24. H1035 - 2605. (top-left) I-band image. The thick contour shows the extent of the galaxy at the Galactic extinctioncorrected I surface brightness of $23 \mathrm{mag} \operatorname{arcsec}^{-2}$. Contour levels are separated by $0.5 \mathrm{mag}$. The H I-dwarf is indicated by the horizontal thick lines. (top-right): $B-I$ map of the galaxy. The overlayed contours correspond to the $B$-band image. (bottom-left) Surface brightness profile in $B$ (filled circles), $I$ (open circles) and $K^{\prime}$ (triangles). An offset of 5 mag has been added to the $K^{\prime}$ SBP. The vertical error bars correspond to $2 \sigma$ uncertainties. The $F W H M$ of point sources in each band is indicated by the vertical lines. (bottom-right) Radially averaged $B-I$ profile

generally show a moderate (if any) luminosity contribution of a younger stellar population superimposed on their LSB components. Very strong emission lines typical of a BCD are associated with the blue compact nucleus. The latter is detected in $K^{\prime}$, at which wavelength another clump is seen $10^{\prime \prime}$ to the South-East without any counterpart in the $I$-band.

- H1034 - 2758: A distorted object with off-centered blue knots. Two galaxies lie at a small projected distance from $\bullet$ H1034 - 2758 (see Fig. 22$):$ G1 $\left(m_{B}=17.5, m_{B}=16.1\right)$ and G2 $\left(m_{B}=18.3, m_{I}=17.3\right)$. The northern object, G2, is a background starburst galaxy at $V_{\text {opt }}=10577 \mathrm{~km} \mathrm{~s}^{-1}$. G1 with $V_{\text {opt }}=3630 \mathrm{~km} \mathrm{~s}^{-1}$ appears as a quiescent dwarf galaxy, a very close interacting companion to H1034-2758 at a projected distance of only $26^{\prime \prime}$ or $5.7 \mathrm{kpc}$. The inner isophotes of H1034 - 2758 seem to be slightly distorted. However, contrary to H1033 - 2642, no signs of truncation in the SBPs profiles are seen in the outer part of the galaxy. The exponential profile of $\mathrm{H} 1034-2758$ reveals an excess in the central core, though less pronounced than in $\mathrm{H} 1034-2558$. The diffuse relatively blue $(B-I \lesssim$ $0.9 \mathrm{mag}$ ) region $\sim 6^{\prime \prime}$ West of the nuclear region corresponds to a strong $\mathrm{H}$ II region.

H1035 - 2756: A peculiar galaxy showing two distinct luminosity knots embedded in a patchy very low-surface brightness $\left(\mu_{B} \gtrsim 25 \mathrm{mag} \operatorname{arcsec}^{-2}\right)$ continuum. Only the eastern clump shows signs of current star formation.

H1035-2605: An elongated face-on object having the reddest integrated color in the sample $(B-I=1.7)$. The galaxy shows two relatively bluer knots at each extremity 

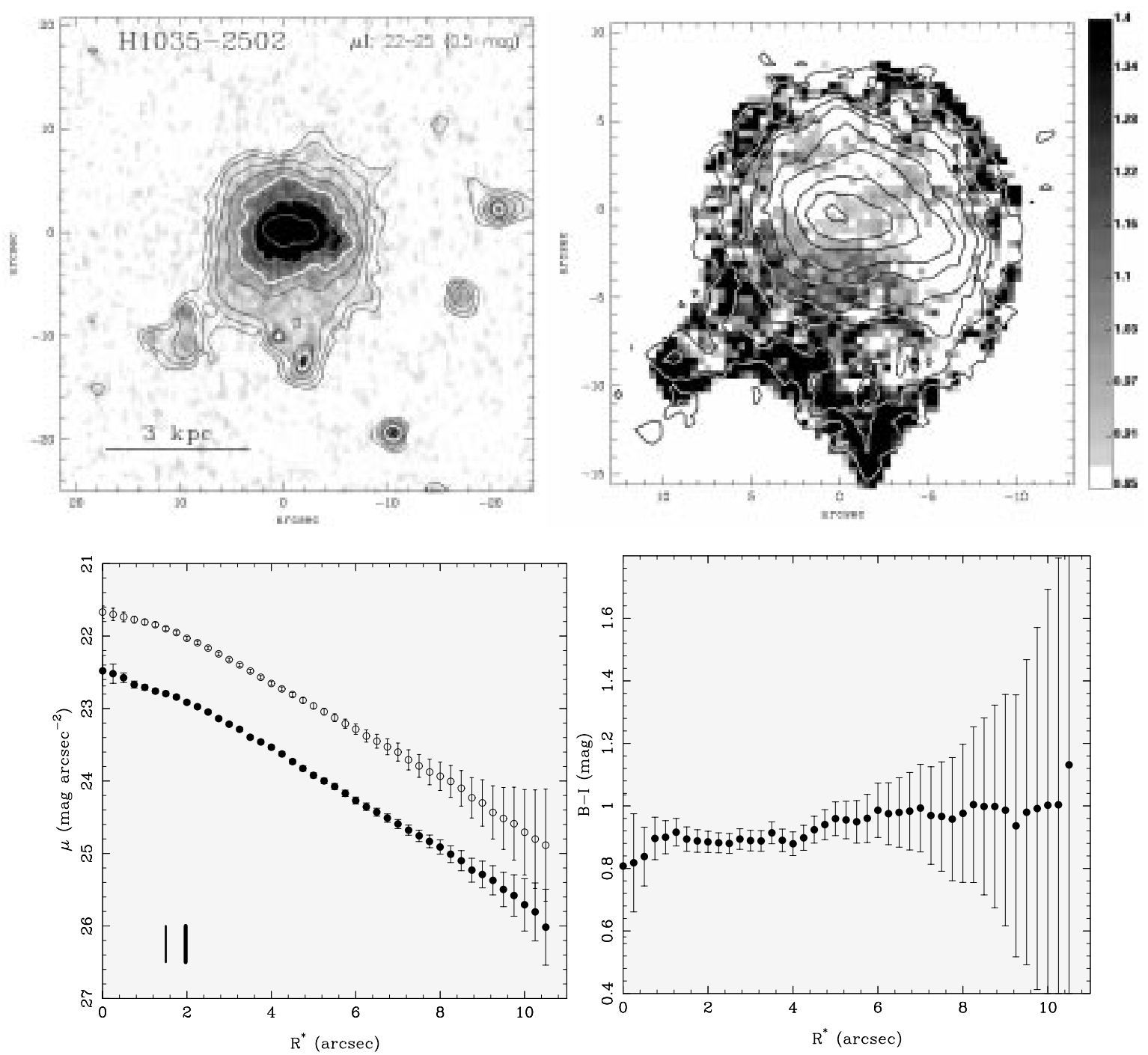

Fig. 25. H1035 - 2502. (top-left) I-band image. The thick contour shows the extent of the galaxy at the Galactic extinctioncorrected I surface brightness of $23 \mathrm{mag} \operatorname{arcsec}^{-2}$. Contour levels are separated by 0.5 mag. (top-right): $B-I$ map of the galaxy. The overlayed contours correspond to the $B$-band image. (bottom-left) Surface brightness profile in $B$ (filled circles) and $I$ (open circles), corrected for Galactic extinction. The vertical error bars correspond to $2 \sigma$ uncertainties. The $F W H M$ of point sources is indicated by the vertical thick and thin lines for $B$ and $I$, respectively. (bottom-right) Radially averaged $B-I$ profile

of its disk with in between a very red region $(B-I \sim$ 2) probably affected by dust extinction. The radially averaged $B-I$ profile shows a negative color gradient of $-0.17 \mathrm{mag} \mathrm{kpc}^{-1}$. The galaxy is well detected in $K^{\prime}$. The $I$ and $K^{\prime}$ peaks occur at the same position. Despite its red color, the optical spectrum indicates an extended starburst. The red nearby objects, designated G1 $\left(m_{B}=18.3\right.$, $\left.m_{I}=16.0\right)$ and $\mathrm{G} 2\left(m_{B}=18.6, m_{I}=16.3\right)$ in Fig. 24 are probably background galaxies, although their optical velocities are yet unknown.

- H1035 - 2502: A blue low-surface brightness object. Extended weak emission lines are observed along a blue bar that shows up in the $B-I$ color map. The Nançay H I spectrum shows a second, narrow peak centered on $V_{\text {hel }}=3620 \mathrm{~km} \mathrm{~s}^{-1}$ with a $F W H M$ of $40 \mathrm{~km} \mathrm{~s}^{-1}$. In the NED and LEDA databases a single large spiral was found in the neighborhood, NGC 3313, whose outer HI disk should be readily detectable at Nançay as its center lies 2'.5 W of the dwarf galaxy (see Fig. 5). H i line observations (VLA imaging by McMahon 1993, Nançay profile by Theureau et al. 1998) show that this face-on 12.4 mag SBb-type spiral is the obvious candidate for the $\mathrm{H}$ I signal, with a central velocity of $3706 \mathrm{~km} \mathrm{~s}^{-1}$, a $F W H M$ of $107 \mathrm{~km} \mathrm{~s}^{-1}$ and a mean flux density of $156 \mathrm{mJy}$.

H1035 - 2740: A relatively blue compact galaxy $(B-I=$ 0.92). This is, with H1034-2558, the only object for which the optical maximum of the galaxy coincides with a blue clump. Emission lines are detected all along the galaxy. The very red object to the North-West, designated as G1 in Fig. 26, was marginally detected in $B\left(m_{B} \sim 20.9\right)$ and 

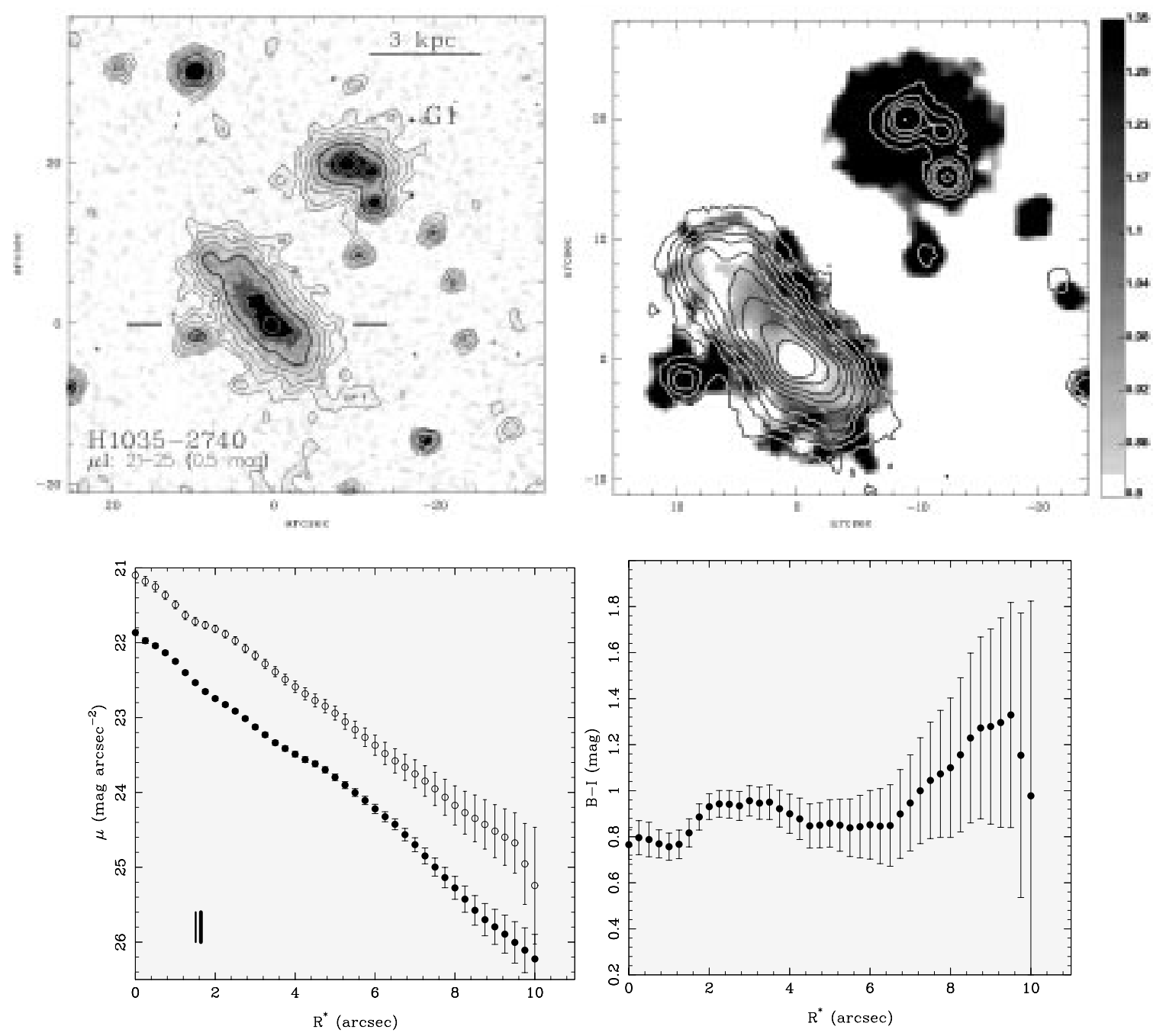

Fig. 26. H1035 - 2740. (top-left) I-band image. The thick contour shows the extent of the galaxy at the Galactic extinctioncorrected I surface brightness of $23 \mathrm{mag} \operatorname{arcsec}^{-2}$. Contour levels are separated by 0.5 mag. (top-right): $B-I$ map of the galaxy. The overlayed contours correspond to the $B$-band image. (bottom-left) Surface brightness profile in $B$ (filled circles) and $I$ (open circles), corrected for Galactic extinction. The vertical error bars correspond to $2 \sigma$ uncertainties. The $F W H M$ of point sources is indicated by the vertical thick and thin lines for $B$ and $I$, respectively. (bottom-right) Radially averaged $B-I$ profile

well seen in $I\left(m_{I} \sim 17.5\right)$. It is most probably a distant background galaxy.

- H1038 - 2733 A low surface galaxy with a bar-like structure. It is the galaxy with the highest H I-mass-to-blue luminosity ratio in the sample $\left(2.3 M_{\odot} / L_{\odot, B}\right)$. The radially averaged $B-I$ profile shows between $\sim 0.2 \mathrm{kpc}$ and $\sim 2.2 \mathrm{kpc}$ radius a weak negative color gradient of -0.12 mag $\mathrm{kpc}^{-1}$. Star formation is observed mainly to the West at the location of a blue region.

The galaxy to the North designated as G1 in Fig. 27 is very red $(B-I \sim 2.7)$ and likely a background galaxy. The Nançay H I spectrum shows a second signal, centered on $V_{\text {hel }}=3975 \mathrm{~km} \mathrm{~s}^{-1}$ with a $W_{20}$ width of $330 \mathrm{~km} \mathrm{~s}^{-1}$. In the NED and LEDA databases a large spiral, NGC 3336, and a smaller pair of galaxies, AM 1038-273, were found in an area 1.5 times the size of the telescope HPBW (see Fig. 8). The $13^{\text {th }}$ mag Sc-type spiral NGC 3336 is the ob- vious candidate for this $\mathrm{H}$ I signal, as shown by the VLA imaging of McMahon (1993) and the Effelsberg detection of Richter \& Huchtmeier (1983), who find a central velocity of $4000 \mathrm{~km} \mathrm{~s}^{-1}$, a $F W H M$ of $300 \mathrm{~km} \mathrm{~s}^{-1}$ and a mean flux density of $57 \mathrm{mJy}$.

H1038 - 2730: An edge-on system with isophote twisting. The radially averaged $B-I$ profile shows a weak negative color gradient of $\sim-0.19$ mag $\mathrm{kpc}^{-1}$. We do not see any evidence of current star-formation occurring in this object. This object has an intriguingly broad $\mathrm{H}$ I line width ( $F W H M 155 \mathrm{~km} \mathrm{~s}^{-1}$ ) given its luminosity and compared to the other edge-on objects in the sample. 

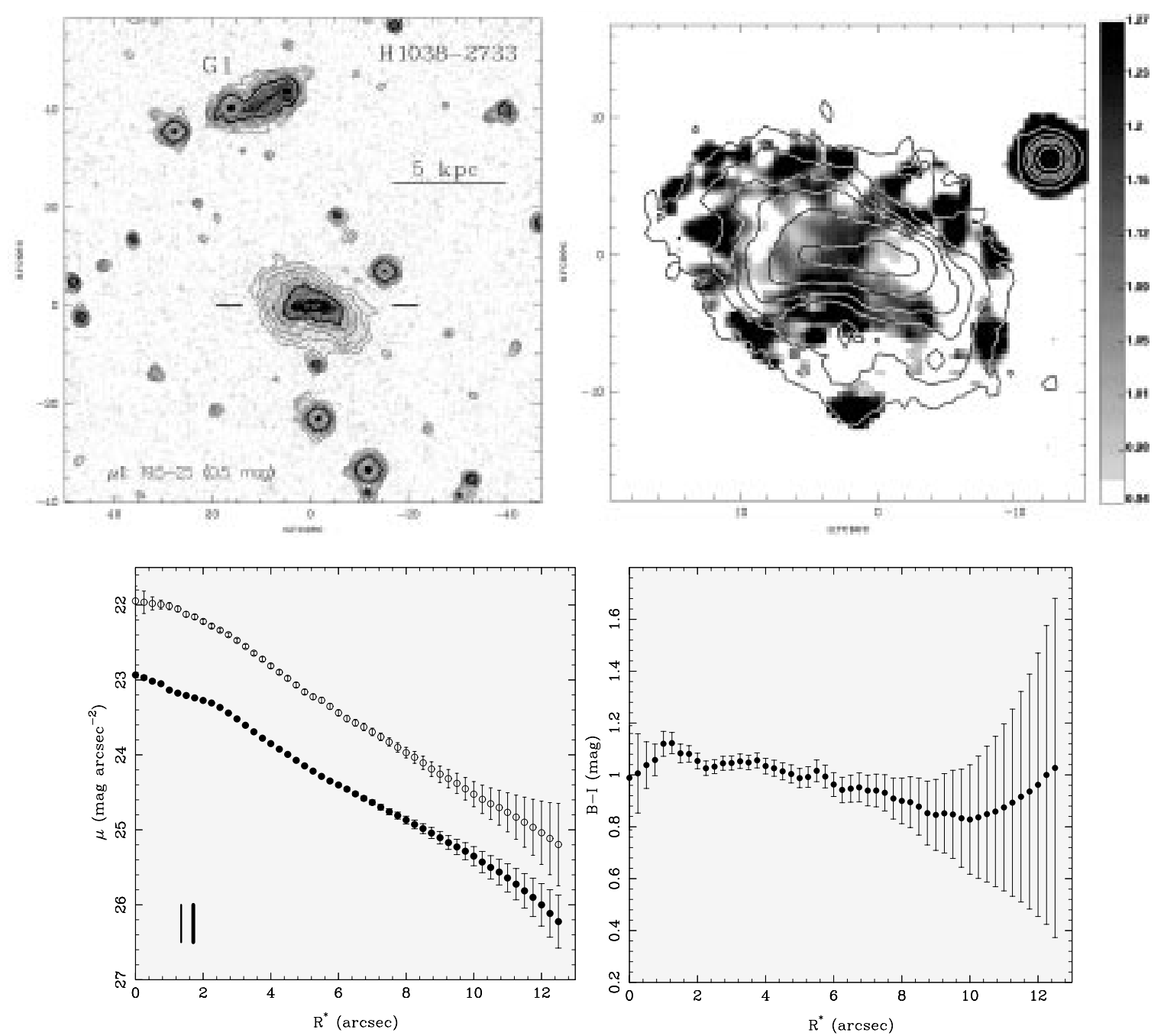

Fig. 27. H1038 - 2733. (top-left) I-band image. The thick contour shows the extent of the galaxy at the Galactic extinctioncorrected I surface brightness of $23 \mathrm{mag} \operatorname{arcsec}^{-2}$. Contour levels are separated by 0.5 mag. (top-right): $B-I$ map of the galaxy. The overlayed contours correspond to the $B$-band image. (bottom-left) Surface brightness profile in $B$ (filled circles) and $I$ (open circles), corrected for Galactic extinction. The vertical error bars correspond to $2 \sigma$ uncertainties. The $F W H M$ of point sources is indicated by the vertical thick and thin lines for $B$ and $I$, respectively. (bottom-right) Radially averaged $B-I$ profile

\section{Conclusions}

We have presented an optical/near-infrared imaging and $\mathrm{HI}$ database for a complete sample of $15 \mathrm{HI}$-selected dwarf galaxies in the Hydra I cluster. The absolute blue magnitude of their optical counterparts ranges between $-15.1 \mathrm{mag}$ and $-17.5 \mathrm{mag}$ and their $\mathrm{H}$ I mass to blue luminosity between 0.1 and $2.4 M_{\odot} / L_{\odot, B}$. Their integrated $B-I$ color index varies between 0.8 and $1.7 \mathrm{mag}$. The low surface brightness components of half of the Hydra dwarfs have an undisturbed exponential profile; the remaining galaxies either show a flattening of their SBP in the central regions or a truncation in the outer regions. One of the H I sources lying in the vicinity of a HI-rich spiral has no optical counterpart.

The heterogeneity of the sample may well be due to the variety of the local environment of each galaxy: in particular the pressure of the intra-cluster medium and the density of the galaxy population. Indeed the dwarfs in Hydra are not uniformly distributed and tend to cluster in substructures, like the more massive galaxies (Valluri et al. 1999). The presence of companions, as commonly observed for the H I-rich dwarfs, may directly affect their morphology and their star formation history. In this paper, we have remarked on these issues on a case-by-case basis only. We will present a general discussion on the role of the environment on the global properties of the Hydra $\mathrm{H} \mathrm{I}-$ rich dwarfs in another article of these series.

Acknowledgements. This work has greatly benefited from discussions with J. van Gorkom. We are grateful to the $2 \mathrm{p} 2$ team at la Silla who helped us with the observations, especially Isabel Peres and Thomas Augusteijn for their efficient and friendly introductions at the D1.5 m and $2.2 \mathrm{~m}$ telescopes. We wish to thank our referee, C. Carignan, for 

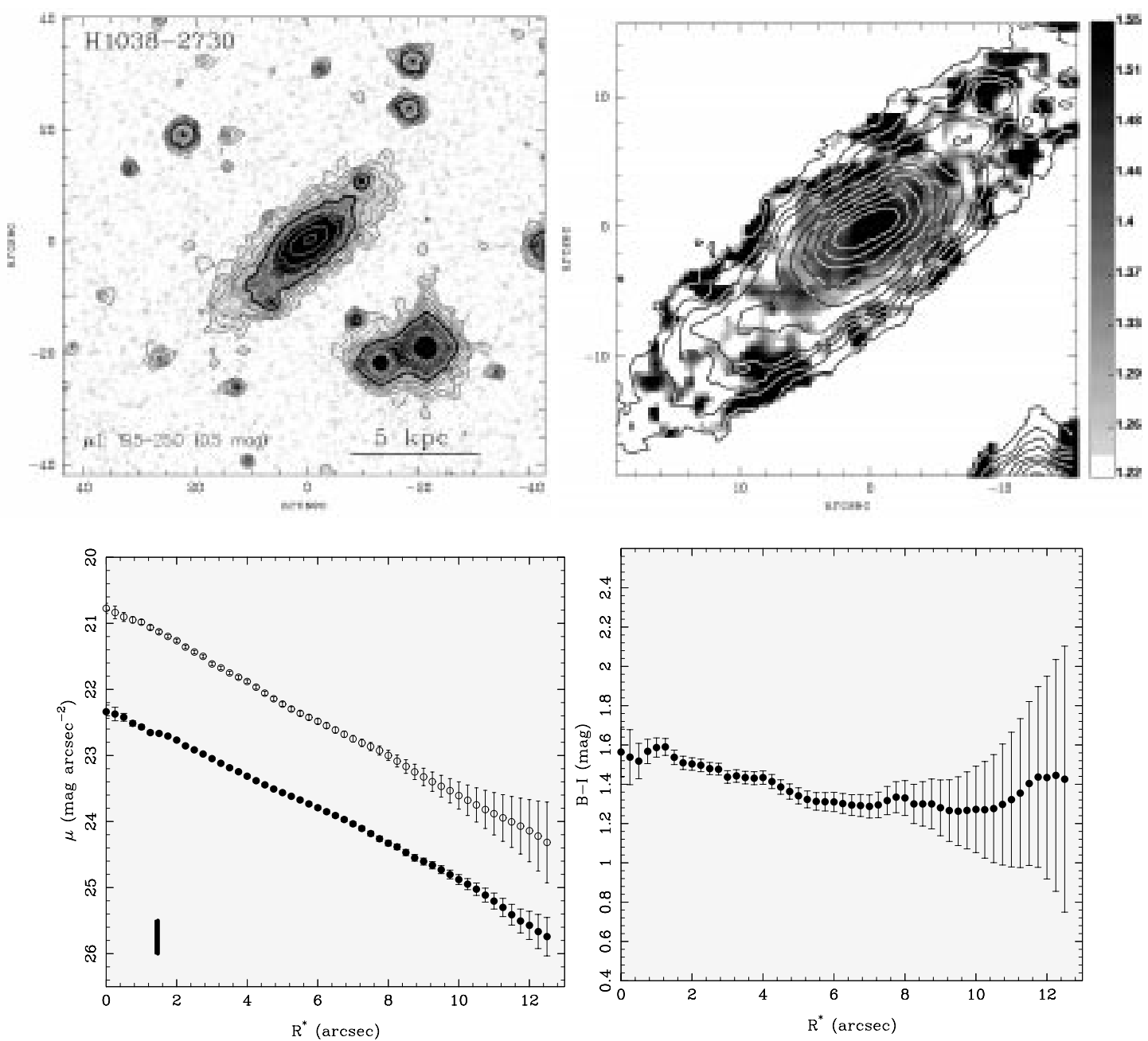

Fig. 28. H1038 - 2730. (top-left) I-band image. The thick contour shows the extent of the galaxy at the Galactic extinctioncorrected I surface brightness of $23 \mathrm{mag} \operatorname{arcsec}^{-2}$. Contour levels are separated by 0.5 mag. (top-right): $B-I$ map of the galaxy. The overlayed contours correspond to the $B$-band image. (bottom-left) Surface brightness profile in $B$ (filled circles) and $I$ (open circles), corrected for Galactic extinction. The vertical error bars correspond to $2 \sigma$ uncertainties. The $F W H M$ of point sources is indicated by the vertical thick and thin lines for $B$ and $I$, respectively. (bottom-right) Radially averaged $B-I$ profile
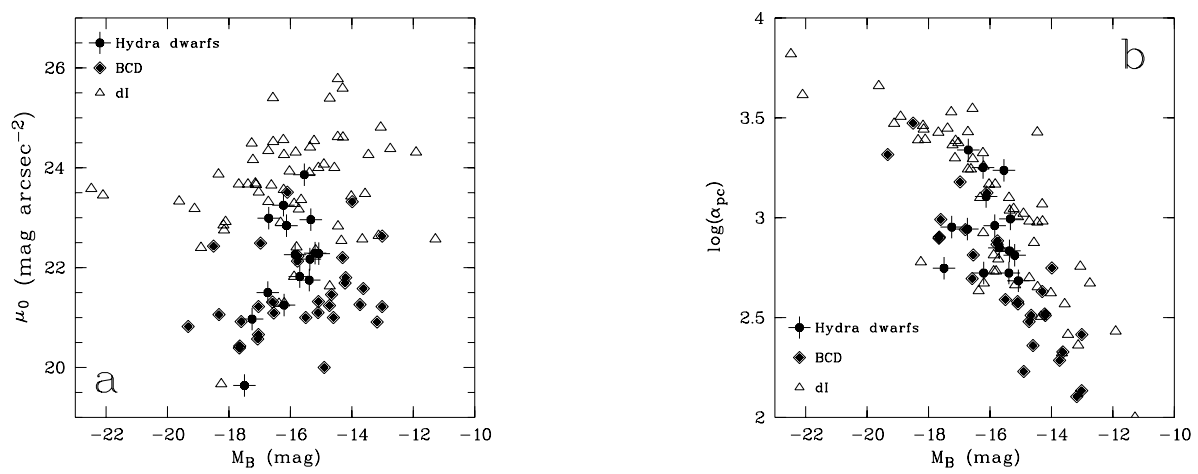

Fig. 29. a) Absolute $B$ luminosity vs. extrapolated central surface brightness of the LSB component for the $\mathrm{H}$ I-detected Hydra dwarfs. For comparison, dwarf irregulars and the LSB component of BCDs from the samples of Patterson \& Thuan (1996) and Papaderos et al. (1996b), respectively, have been added. Note that roughly $1 / 3$ of the surface brightness profiles of the $\mathrm{H}$ I-detected d warfs belong to the type-V class; e.g. they exhibit an outer exponential slope and a flattening towards the center. In these cases, the extrapolated central surface brightness $\mu_{0}$ may differ significantly from the value actually observed at the center of the galaxy. b) Absolute B luminosity vs. exponential scale length. All quantities shown for the Hydra dwarfs are corrected for foreground Galactic extinction 
Table 5. Integrated properties

\begin{tabular}{|c|c|c|c|c|}
\hline Name & $\begin{array}{c}M_{B} \\
\operatorname{mag} \\
(2)\end{array}$ & $\begin{array}{c}M_{\mathrm{HI}} \\
10^{8} M_{\odot} \\
(3)\end{array}$ & $\begin{array}{c}M_{\mathrm{HI}} / L_{B} \\
M_{\odot} / L_{\odot, B} \\
\quad(4)\end{array}$ & $\begin{array}{c}B-I \\
\operatorname{mag} \\
(5)\end{array}$ \\
\hline H1031 - 2818 & -17.50 & 2.3 & 0.16 & 1.57 \\
\hline H1031 - 2632 & -16.13 & 1.8 & 0.40 & 1.49 \\
\hline H1032 - 2638 & -15.20 & (1.8) & 0.97 & 1.17 \\
\hline H1032 - 2722 & -15.70 & 4.3 & 1.46 & 1.02 \\
\hline H1033 - 2707 & -16.21 & 5.7 & 1.22 & 0.97 \\
\hline H1033 - 2642 & -16.71 & 5.2 & 0.71 & 1.25 \\
\hline H1033 - 2722 & -16.23 & 7.3 & 1.52 & 1.27 \\
\hline H1034 - 2558 & -15.08 & (1.1) & 0.69 & 0.76 \\
\hline H1034 - 2758 & -17.25 & 4.7 & 0.39 & 1.10 \\
\hline H1035 - 2756 & -15.55 & 6.2 & 2.40 & 1.42 \\
\hline H1035 - 2605 & -16.74 & $(0.7)$ & 0.10 & 1.69 \\
\hline H1035 - 2502 & -15.37 & 2.4 & 1.10 & 0.93 \\
\hline H1035 - 2740 & -15.39 & $(1.5)$ & 0.67 & 0.92 \\
\hline H1038 - 2733 & -15.34 & 5.0 & 2.34 & 0.96 \\
\hline H1038 - 2730 & -15.85 & 6.1 & 1.80 & 1.38 \\
\hline H1031 - 2734 & -17.70 & 10.1 & 0.54 & 1.32 \\
\hline H1032 - 2819 & - & 4.6 & - & - \\
\hline H1033 - 2726 & -17.36 & 9.3 & 0.68 & 1.11 \\
\hline H1033 - 2506 & -19.00 & 9.5 & 0.15 & 1.53 \\
\hline H1034 - 2553 & -18.04 & 18.3 & 0.72 & 1.39 \\
\hline
\end{tabular}

valuable comments. Research by P.P. has been supported by Deutsche Agentur für Raumfahrtangelegenheiten (DARA) GmbH grant 50 OR 9407 6. The Unité Scientifique Nançay of the Observatoire de Paris is associated as USR B704 to the French Centre National de Recherche Scientifique (CNRS). Nançay also gratefully acknowledges the financial support of the Région Centre in France. DAEC, the Department of Extragalactic Astronomy and Cosmology of the Observatoire de Paris is associated with the Université de Paris 7 and as URA 173 to the CNRS. This research has made use of the Lyon-Meudon Extragalactic Database (LEDA) supplied by the LEDA team at the CRAL-Observatoire de Lyon (France), as well as of the NASA/IPAC Extragalactic Database (NED) which is operated by the Jet Propulsion Laboratory, California Institute of Technology, under contract with the National Aeronautics and Space Administration.

\section{References}

Almoznino E., Brosch N., 1998, MNRAS 298, 931

Bautz L.P., Morgan W.W., 1970, ApJ 162, L149

Binggeli B., Cameron L.M., 1991, A\&A 252, 27

Bothun G.D., Mould J.R., Caldwell N., Macgillivray H.T., 1986, AJ 92, 1007

Bravo H., Cayatte V., van Gorkom J., Balkowski C., 1999 (in preparation)
Cayatte V., van Gorkom J.H., Balkowski C., Kotanyi C., 1990, AJ 100, 604

Dickey J.M., 1997, AJ 113, 1939

Dressler A., Oemler A.J., Couch W.J., et al., 1997, ApJ 490, 577

Fitchett M., Merritt D., 1988, ApJ 335, 18

Fukunaga-Nakamura A., Tosa M., 1989, PASP 41, 953

Gallagher J.S. III, Hunter D.A., 1989, AJ 98, 806

Hamabe M., 1993, ApJS 85, 249

Hoffman G.L., Helou G., Salpeter E.E., 1988, ApJ 324, 75

Hoffman G.L., Salpeter E.E., Farhat B., Roos T., Williams H., Helou G., 1996, ApJS 105, 269

Impey C., Bothun G., Malin D., 1988, ApJ 330, 634

James P., 1991, MNRAS 250, 544

Kenney J.D.P., 1990, in The interstellar Medium in Galaxies, Thronson H.A., Shull M. (eds.). Kluwer Academic Publishers, p. 151

Landolt A.U., 1992, AJ 104, 340

Matthews L.D., van Driel W., Gallagher J.S. III, 1998, AJ 116, 1169

Maza J., Ruiz M.T., Gonzalez L.E., Wischnjewsky M., Peña M., 1991, A\&AS 89, 389

McMahon P.M., 1993, Ph.D. thesis. Columbia University

McMahon P.M., Van Gorkom J.H., Richter O.G., Ferguson H.C., 1992, AJ 103, 399

Papaderos P., Loose H.H., Fricke K.J., Thuan T.X., 1996a, A\&A 314, 59

Papaderos P., Loose H.H., Thuan T.X., Fricke K.J., 1996b, A\&AS 120, 207

Patterson R.J., Thuan T.X., 1996, ApJS 107, 103

Paturel G., Fouqué P., Bottinelli L., Gouguenheim L., 1989, Catalogue of Principal Galaxies. Observatoires de Lyon et de Paris-Meudon

Peña M., Ruiz M.T., Maza J., 1991, A\&A 251, 417

Persson S.E., Murphy D.C., Krzeminski W., Roth M., Rieke M.J., 1998, AJ 116, 2475

Richter O.-G., 1987, A\&AS 67, 237

Richter O.-G., 1989, A\&AS 77, 237

Richter O.-G., Huchtmeier W.K., 1983, A\&A 125, 187

Richter O.-G., Huchtmeier W.K., 1987, A\&AS 68, 427

Richter O.-G., Huchtmeier W.K., Materne J., 1982, A\&A 111, 193

Schweizer F., 1998, in Galaxies: interactions and induced star formation Kennicutt R., Schweizer F., Barnes J. (eds.). Springer

Secker J., Harris W.E., Plummer J.D., 1997, PASP 109, 1377

Skillman E., 1996, in The Minnesota Lectures on Extragalactic Neutral Hydrogen Skillman E. (ed.), ASP Conf. Ser. 106, 208

Theureau G., Bottinelli L., Coudreau-Durand N., et al., 1998, A\&AS 130, 333

Thuan T.X., 1985, ApJ 299, 881

Trentham N., 1998, MNRAS 293, 71

Valluri M., Jog C.J., 1990, ApJ 357, 367

Valluri M., van Gorkom J., McMahon P., 1999, AJ (submitted) 